\title{
THE ROMAN DENARII HOARD IN THE ERIMTAN COLLECTION
}

\author{
MELİH ARSLAN - ÜLKÜ DEVECİOĞLU
}

The hoard is in the collection of Mr. Yüksel Erimtan who is a private collector of ancient cultural objects registered at the Anatolian Civilizations Museum in Ankara. The hoard which consists of 246 silver Roman coins was purchased in 1993 and registered in the inventory book. The 243 of 246 coins are denarii and three of them are antoninianii (see catalogue no. 239, 242, and 244). Those three antoninianii belong to Elagabalus, the last emperor represented in the treasure.

The hoard fundamentally encompasses the reigns of 13 emperors (see. Table I). The coins in the treasure were struck in the name of 20 different individuals (emperor, empress and caesar) with their likenesses. Some emperors were also represented when they had been heirs to the throne (caesar). So there are a total of 23 groups as emperors, caesars and empresses in the treasure (see Table II). The catalogue here shows the coins chronologically listed depending on the Table II. As it is evident in the Table II the Erimtan Denarii Hoard exhibits a very homogenous entity embracing the coins of 20 individuals from 13 reigns. From Vespasian to Elagabalus all emperors are chronologically represented. Only the coins of Pertinax, Didius Julianus and Pescennius Niger, short term emperors of the Severan period lack in this treasure. However, one emperor from the same period Clodius Albinus is represented with two coins (Cat. No. 97-98).

Beside denarii there are only three antoninianii in the treasure. The emperor Caracalla introduced this new silver denomination valued 2 Denarii for the first time in A.D. 215. The new denomination was named antoninianus after the Emperor Caracalla's official name Marcus Aurelius Antoninus. The antoninianii were in use until the reign of the Emperor Diocletianus (A.D. 284-305). Although the antoninianii were projected to be heavier and larger than their predecessors their silver content was less than the denarii and in time silver in those coins continued to decrease until eventually it remained as a silver wash on the surface of bronze coins.

The denarius, the fundamental denomination of Rome was first issued during the Roman Republic in 211 B.C. This denomination was continued to be used until the end of the Roman Empire. Although they weighed approximately 4.00 grams in the beginning they were drastically decayed both in weight and silver content toward the end of the imperial times due to weakening of the Roman Empire and the economic failure.

The Erimtan hoard comprises the time period from the reign of Vespasianus (A.D. 6979) to Elagabalus (A.D. 218-222). The change in the standard weight of the denarii can be observed in this treasure of coins that span a period of about 150 years. 
The hoard coins belong to three different dynasties in the Roman Empire. In the first group are the coins of the Flavian dynasty emperors (Vespasianus, Titus and Domitianus). However, the coins of Titus and Domitianus were struck in the reign of their father Vespasianus while they were caesars. This is the least crowded group of the hoard.

The second group encompasses the Antonine dynasty emperors. The coins of Nerva, Trajan, Hadrian, Antoninus Pius, Marcus Aurelius, Lucius Verus, Commodus and their families are in the hoard. Commodus (33 coins) and Antoninus Pius (14 coins) are represented with the most coins among the Antonine dynasty emperors.

The third and the last group contains the coins of the Severan dynasty emperors including Septimius Severus, Clodius Albinus, Caracalla, Geta, Elagabalus and their families. While Caracalla is represented both with his caesar and augustus period coins, Geta is only represented as caesar. There are the coins of empresses such as Julia Domna and Plautialla in this group. The most coins in the treasure are in this group. Furthermore emperors and empress with the most coins Septimius Severus (52 coins), Caracalla (37 coins) and Julia Domna (28 coins) are also in this group.

So, while the Severans are represented with the most coins, Antonines were represented with fewer and finally the Flavians represented with the fewest coins.

The 243 denarii and the 3 antoninianii of the treasure embrace a time period of 150 years from Vespasian to Elagabalus. The Elagabalus' coins of A.D. 222 from the Rome mint (cat. No. 241-245) and the coin of the same year from the Antiochia mint (cat. no. 246) are the terminus post quem of the hoard. The treasure must have been buried after that period.

The great majority of the coins of the hoard are from the mint of Rome. It is observed that very few coins come from the eastern - Syrian mints (Antiochia, Emesa and Laodiceia). While there are only single examples from the mints of Antiochia (no. 246), and Emesa (no. 146), there are 9 examples from the Laodiceia (no. 147-150; 178; 212-215) mint. All of the 1 lcoins from the eastern mints belong to the Severan dynasty period emperors (Septimius Severus, Caracalla ve Elagabalus). It is known that those eastern mint coins were struck to pay the mercenaries in the Parthian campaign.

In terms of burial date the Erimtan Hoard is consistent with the other contemporary Asia Minor hoards both in homogeneousness and chronology. The first of the similar denarii hoards is known as the Haydere (Aydın-Bozdoğan Treasure-1982). The original number of coins in the Haydere hoard is 5578 and 2330 of them are in the İzmir Museum. The lot in the İzmir Museum consists of 1113 denarii and 1216 antoninianii and they were first published by P. Aydemir and his friends ${ }^{1}$. The Haydere Hoard encompasses a time period of 200 years from Vespasian to Gallienus. The coins from this hoard, except the antoninianii of the Severan period have a lot in common with the Erimtan Denarii Hoard. Another similar treasure is the one that was unearthed in the Koçoğlu village of Manyas Township of Balıkesir province in 1985. The court documents indicated that there were 2469 coins in the treasure when it was first discovered. However only 210 of those coins were seized and surrendered to 
the Balıkesir Museum. The whereabouts of the rest of the coins is unknown. The 210 coins from that hoard were brought to the Anatolian Civilizations Museum for inspection in 1987 and they still remain there. The 208 of the 210 coins in the Manyas Hoard are denarii and also two are drachms minted in Amisus. The treasure spans a time period of 100 years from the emperor Otho to Antoninus Pius. The Manyas Denarii Treasure was published by this author in $1996^{2}$. In terms of content this treasure resembles the Erimtan Treasure. There are two more denarius hoards by the names the Sulakyurt Treasure and the Yatağan Treasure in the Anatolian Civilizations Museum. The Yatağan Hoard is registered with the inventory number 1861 in the museum. 1559 is the inventory number for the Sulakyurt Treasure. There are 243 denarii in the Yatağan Hoard while the Sulakyurt Hoard consists of 429 denarii. The earliest coins in both treasures are from the reign of Antoninus Pius. The Yatağan Hoard coins ranges from Antoninus Pius (A.D. 138-161) to Gordianus III (A.D. 238-244) and the Sulakyurt coins come to an end with the coins of Maximinus I (235-238). Those two hoards that exhibit great similarities to the Erimtan Hoard were published twice by Ms. Yildız Kizılkaya ${ }^{3}$.

Another similar denarius hoard to the Erimtan one has recently been discovered in the vicinity of the Kurkkale province. The 110 denarii from that hoard were purchased by the Anatolian Civilizations Museum and they were registered with the inventory numbers 71110-99. Later, I found out that the rest of the lot which constituted the great majority of the hoard was sold to the Ereğli (the Black Sea region) Museum. It is said that approximately 500 to 600 of the Ereğli coins are denarii. The 110 coins part of the Kırıkkale Hoard in the Anatolian Civilizations Museum begins with Emperor Otho (A.D. 69) and ends with Emperor Commodus (A.D. 177-192). This hoard also spans a time period of 150 years as in the example of the Erimtan Hoard ${ }^{4}$. A hoard of 13 Roman silver coins was unearthed during the 1977 excavations of ancient Erythrai and it was published ${ }^{5}$. The Silver Erythrai Hoard consists of the coins of Domitian to Septimius Severus, a time span of 100 years. Three of those coins are in the cistophor denomination and 10 of them are denarii. The Erythrai Hoard coins are contemporary and similar to the coins of the Erimtan ones. The 2306 silver coin hoard found in Viuz-Faverges, Alta Savoia, France contains 1780 denarii, 525 antoninianii and 1 Amisus drachm. The French Hoard embraces the time period from Nero (A.D. 64) to Maximinus (A.D. 235) ${ }^{6}$. This large silver coin hoard found outside of Anatolia is contemporary of and it carries similarities with the Erimtan coins in terms of denarii.

There are a few other published hoards that are less contemporary of the Erimtan Hoard. One of those, which was found in the Agora excavation of ancient Iasos begins with coins of Caracalla and continues until the reign of Gallienus. Only 11 of the 2997 coins are denarii while the 2986 are antoninianii?

See Arslan 1996: 31-36.

See Kızılkaya 1981: 103-134 ve Kızılkaya 1991: 213-248 and Kızılkaya 1988: 137-169 ve Kızılkaya 1991a: 249-273.

The 110 coins part of the Kirikkale Treasure is going to be published by myself.

See Pasinli 1980: 95-110.

See H. G. Pflaum-H. Huvelin, "Le Trésor Viuz-Faverges". Trésor Monétaires III (1981): 33-38.

See Tondo 2005: 29-74. 
Metrology: The coin with the least weight in the Erimtan Denarii Hoard is the one of Commodus weighing 2.26 grams (see no. 76). The last coin in the catalog (no. 246) weighs 3.91 grams and it is also the heaviest denarius coin in the treasure. Except this coin of Elagabalus there are two more rather heavy denarii in the hoard. Both of those two coins weigh 3.82 grams (see no. 133 and 192). The coin number 133 belongs to Septimius Severus and the number 192 belongs to Caracalla. Furthermore the three antoninianii in their own denomination are the heaviest coins in the hoard. The number 239 coin is 4.17 grams and the number 242 coin is 5.56 grams while the number 244 coin weighs 4.95 grams. In general the majority of the coins in the hoard are over 3.00 grams each. The weights of 35 of the coins range from 3.50 grams to 3.91 grams. Furthermore the weights of 43 coins change between 2.26 grams and 2.99 grams. The total weight of the hoard is 800.54 grams and the average coin weight is 3.25 grams. In general the coins of the hoard are well preserved and they don't seem to have worn out in circulation.

The three emperors with the most coins are predominant in the hoard: Septimius Severus (52 coins), Caracalla (37 coins) and Commodus (31 coins). Separately Julia Domna also has 28 coins. When the coin weights of those three emperors are compared, one observes that the majority of the coins of Commodus are less than 3.00 grams. The 19 of the 31 Commodus denarii are under 3.00 grams. The heaviest of the Commodus coins is 3.57 grams. The majority coins in the Septimius Severus group of 52 denarii are more than 3.00 grams. Only a small part of the 52 coins, 11 of them are lees than 3.00 grams. The denarius that weighs the least among the Septimius Severus coins is 2.49 grams. The Caracalla coins are better preserved than the others and most coins are heavier than 3.00 grams. Only 5 of the 37 Caracalla coins weigh less than 3.00 grams. Only 2 of the 28 Julia Domna coins are lighter than 3.00 grams while all the others have an average weight of approximately 3.40 grams and they are very well preserved.

Die Connections: The obverse and reverse die connections among the coins of the hoard have also been investigated. It was found that some examples come from the same dies. All of the same dies are obverse dies. Although there are some very similar ones no die connections could be determined among the reverses. The coins that come from the same obverse dies are as follows:

1-) number 85 and 86 Commodus coins.

2-) number 116 and 117 Septimius Severus coins.

3-) number 131 and 133 Septimius Severus coins.

4-) number 181 and 182 Caracalla Caesar coins.

5-) number 218 and 219 Plautilla coins.

Conclusion and Assessment: The Erimtan silver Roman coins hoard that consists of 243 denarii and 3 antoninianii was unearthed in 1993. Although the find location is not known it is almost certain that it was found in Anatolia. We also don't have any information about the original content of the hoard but it is possible that it might be a bigger hoard 
because as experience proved larger treasures like this are generally sold after they are partitioned.

Even though the great majority of the coins of the Erimtan Denarii Hoard are known types the Faustina II coin (cat. No.59) is not seen in the present publications. Cohen and RIC catalogues only have the gold aureus variations of the same type. In addition, the number 128 coin of Septimius Severus with IVNO REGINA legend is also a new type.

It is observed that while most coins in the hoard come from the Rome mint there is a certain number of examples from the so called eastern mints, namely Antiochia, Emesa ve Laodiceia ad Mare of Syria. Especially the Syrian mint coins of the latest emperor in the treasure indicate the latest time of use of the hoard coins and their relationship with the conflict between Rome and Parthia. To finance the Parthian campaigns Romans mostly depended on coins produced in the Syrian mints as well as the mint of Rome.

The coins of the hoard represent consecutive emperors and span a time period of approximately 150 years. The Erimtan Denarii Hoard in its present entirety might be a coin collection of a person or a personal accumulation as well as a collected sum of a tax office. Regardless of its intention it is a very important development that such a valuable hoard found by chance is recorded in the collection of a respected collector. Without the initiative of Mr. Yüksel Erimtan who always adores ancient art works and supports scientific research it would be impossible to publish this valuable hoard in its entirety. 


\section{Gatalogue}

\section{Vespasian (A.D. 69-79)}

Mint of Rome (A.D. 73)

1. Obv. IMP. CAES. VESP. AVG. P. M. COS. IIII. CEN.

Head of Vespasian, laureate, $r$.

Rev. FIDES PVBL.

Clasped hands holding caduceus, poppies and corn-ears.

Inv. No. $\quad 106$

$S / W / D \quad 18 \mathrm{~mm}$; $3.09 \mathrm{gr}$; $6 \mathrm{~h}$.

Ref. Cohen, 163; BMCRE, 86; RIC, 55.

\section{Mint of Rome (A.D. 74)}

2. $O b v$. IMP. CAESAR VESP. A[VG.]

Head of Vespasian, laureate, $r$.

Rev. $\quad$ COS. V.

Two laurel-branches.

Inv. No. 104

$S / W / D \quad 19 \mathrm{~mm} . ; 3.16 \mathrm{gr}$; $5 \mathrm{~h}$.

Ref. Cohen, 110; BMCRE, 133; RIC, 72.

Titus, As Caesar (A.D. 69-79)

Mint of Rome (A.D. 75)

3. $O b v$. CAESA[R] IMP. VESPASIAN

Head of Titus, laureate, $r$.

Rev. PONTIF. TR. P. COS. IIII.

Pax seated l., holding branch.

Inv. No. 107

$S / W / D \quad 19 \mathrm{~mm}$; 3.17 gr.; $7 \mathrm{~h}$.

Ref. Cohen, 162; BMCRE, 172; RIC, 185.

Titus Augustus (A.D. 79-81)

Mint of Rome (A.D. 79-80)

4. $O b v$. IMP. TITVS CAES. VESPASIAN. AVG. P. M.

Head of Titus, laureate, $r$.

Rev. [TR. P. IX. IMP.] XV. COS. VIII. P. P.

Throne with round back, on which are corn-ears.

Inv. No. 105

$S / W / D \quad 18 \mathrm{~mm}$.; 3.17 gr.; $6 \mathrm{~h}$.

Ref. Cohen, 313; BMCRE, 58; RIC, 24a. 


\section{Mint of Rome (A.D. 80-81)}

5. Obv. DIVVS AVGVSTVS VESPASIANVS

Head of Vespasian, laureate, $r$.

Rev. $\quad$ EX. [SC].

Shield, resting against cippus, on which stands an urn: laurels $\mathrm{r}$. and $\mathrm{l}$.

Inv. No. 103

$S / W / D \quad 18 \mathrm{~mm}$.; $3.30 \mathrm{gr}$; $7 \mathrm{~h}$.

Ref. Cohen, 149; BMCRE, 125; RIC, 62.

Domitian, As Caesar (A.D. 69-79)

Mint of Rome (A.D. 73)

6. Obv. CAES. AVG. F. DOMIT. COS. II.

Head of Domitian, laureate, $r$.

Rev. $\quad$ No legend.

Domitian riding l., raising $\mathrm{r}$. hand and holding sceptre with human head in 1 . hand.

Inv. No. 108

$S / W / D \quad 19$ mm.; 3.13 gr.; $12 \mathrm{~h}$.

Ref. $\quad$ Cohen, 664; BMCRE, 129; RIC, 232.

Nerva (A.D. 96-98)

Mint of Rome (A.D. 97)

7. Obv. IMP. NERVA CAES. AVG. P. M. TR. P. COS. III. P. P.

Head of Nerva, laureate, $r$.

Rev. [CO]NCORDIA EXERCITVVM

Clasped hands, holding legionary eagle, resting on prow.

Inv. No. 109

$S / W / D \quad 18 \mathrm{~mm}$.; 3.14 gr.; $6 \mathrm{~h}$.

Ref. Cohen, 29; BMCRE, 29; RIC, 15.

8. Obv. IMP. NERVA CAES. AVG. P. M. TR. P. II. COS.

III. P. P.

Head of Nerva, laureate, $r$.

Rev. AEQVITAS AVGVST.

Aequitas standing 1., holding scales and cornucopiae.

Inv. No. $\quad 110$

$S / W / D \quad 18 \mathrm{~mm}$; 3.23 gr.; $6 \mathrm{~h}$.

Ref. Cohen, 9; BMCRE, 52; RIC, 25.

Trajan (A.D. 98-117)

Mint of Rome (A.D. 98-99)

9. Obv. IMP. CAES. NERVA TRA[IAN] AVG. GERM. 


$\begin{array}{ll} & \text { Head of Trajan, laureate, r. } \\ \text { Rev. } & \text { P. M. TR. P. COS. II. P. P. } \\ & \text { Vesta seated I., holding patera and torch. } \\ \text { Inv. No. } & 115 \\ S / W / D & 19 \mathrm{~mm} . ; 3.33 \mathrm{gr} . ; \text { 7h. } \\ \text { Ref. } & \text { Cohen, 203; BMCRE, 26; RIC, 9. }\end{array}$

10. Obv. IMP. CAES. NERVA TRAIAN AVG. GERM.

Head of Trajan, laureate, $r$.

Rev. $\quad$ PONT. MAX. TR. POT. COS. II.

Victory seated l., holding patera and palm.

Inv. No. 116

$S / W / D \quad 18 \mathrm{~mm}$; 2.86 gr.; $6 \mathrm{~h}$.

Ref. Cohen, 295; BMCRE, 21; RIC, 22.

\section{Mint of Rome (A.D. 101-102)}

11. $O b v$. IMP. CAES. NERVA TRAIAN AVG. GERM.

Head of Trajan, laureate, $r$.

Rev. P. M. TR. P. COS. IIII. P. P.

Victory walking r.,holding wreath and palm.

Inv. No. 112

$S / W / D \quad 19 \mathrm{~mm}$; 3.05 gr.; $7 \mathrm{~h}$.

Ref. Cohen, 242; BMCRE, 121; RIC, 60.

\section{Mint of Rome (A.D. 103-111)}

12. Obv. IMP. [TRAIA]NO AVG. GER. DAC. P. M. TR. P.

Head of Trajan, laureate, $r$.

Rev. DAC. CAP. COS. V. P. [P. S.P.Q.R. OPTI]MO PRINC.

Dacian seated., mourning; various arms on ground.

Inv. No. 113

$S / W / D \quad 19 \mathrm{~mm}$.; $3.20 \mathrm{gr}$; $7 \mathrm{~h}$.

Ref. Cohen, 120; BMCRE, 390; RIC, 98.

13. Obv. IMP. TRAIANO AVG. GER. DAC. P. M. TR. P.

Bust of Trajan, laureate, $r$.

Rev. COS. V. P. P. S.P.Q.R.OPTIMO PRINC

Aequitas seated 1., holding scales and cornucopiae.

Inv. No. 114

$S / W / D \quad 19$ mm.; 3.07 gr.; $6 \mathrm{~h}$.

Ref. Cohen, 86; BMCRE, 288; RIC, 119. 
14. Obv. IMP. TRAIANO AVG. GER. DAC. P. M. TR. P.

Head of Trajan, laureate, $r$.

Rev. $\quad$ COS. V. P. P. S. P. Q. R. OPTIMO PRINC.

Spes, draped, advancing l., holding up flower in $\mathrm{r}$. hand and lifting up skirt with 1 .

Inv. No. 111

$S / W / D \quad 19 \mathrm{~mm}$; 3.12 gr.; $6 \mathrm{~h}$.

Ref. $\quad$ Cohen, 84 (reverse); BMCRE, 319; RIC, 127.

15. Obv. IMP. CAES. NER. TRAIAN OPTIM. AVG. GER. DAC.

Bust of Trajan, laureate, draped, $r$.

Rev. $\quad$ FORT. RED. [PARTHICO P. M. T]R. P. COS. VI. P. P. S. P. Q. R.

Fortuna seated 1 , holding rudder and cornucopiae.

Inv. No. 117

$S / W / D \quad 19 \mathrm{~mm}$; $3.21 \mathrm{gr}$; $6 \mathrm{~h}$.

Ref. Cohen, 150; BMCRE, 634; RIC, 316.

Hadrian (A.D. 117-138)

Mint of Rome (A.D. 119-122)

16. Obv. [IMP. CAESA]R TRAIAN HADRIANVS AVG.

Head of Hadrian, laureate, $r$.

Rev. $\quad$ P. M. TR. P. COS. III. $\square$

Fortuna standing 1.,holding rudder on ground in $r$. hand and cornucopiae in $1 ., 1$. arm resting on column.

Inv. No. 118

$S / W / D \quad 19$ mm.; 3.18 gr.; $6 \mathrm{~h}$.

Ref. Cohen, 1155; BMCRE, 174; RIC, 86.

Mint of Rome (A.D. 125-128)

17. Obv. HADRIANVS AVGVSTVS

Head of Hadrian, laureate, $r$.

Rev. COS. III.

Neptün standing l.; r. foot on prow, holding trident and acrostolium.

Inv. No. 119

$S / W / D \quad 18 \mathrm{~mm}$; $3.23 \mathrm{gr}$; $7 \mathrm{~h}$.

Ref. $\quad$ Cohen, 309; BMCRE, 354; RIC, 158.

18. Obv. HADRIANVS AVGVSTVS

Head of Hadrian, laureate, $r$.

Rev. COS. III.

Concordia seated 1., holding patera, elbow resting on figure of Spes.

(Occasionally there is a cornucopiae below the chair). 
Inv. No. 123

$S / W / D \quad 19 \mathrm{~mm}$.; $3,22 \mathrm{gr}$; $6 \mathrm{~h}$.

Ref. $\quad$ Cohen, 328; BMCRE, 391; RIC, 172.

\section{Mint of Rome (A.D. 134-138)}

19. Obv. HADRIANVS AVG. COS. III. P. P.

Head of Hadrian, bare, $r$.

Rev. FORTVNAE REDVCI.

Fortuna standing 1., holding cornucopiae and clasping $r$. hands with Hadrian standing r.; Fortuna rests on a rudder set on globe.

Inv. No. 120

$S / W / D \quad 17$ mm.; 3,23 gr.; $6 \mathrm{~h}$.

Ref. $\quad$ Cohen, 788; BMCRE, 650; RIC, 248.

20.

\begin{tabular}{ll}
\multicolumn{2}{c}{ Similar } \\
Inv. No. & 121 \\
S/W/D & $17 \mathrm{~mm} . ;$ \\
Ref. & Cohen, 788; BM.; $6 \mathrm{~h}$. \\
\hline
\end{tabular}

21. Obv. HADRIANVS AVG.COS. III. P.P.

Head of Hadrian, laureate, $r$.

Rev. $\quad$ VOTA PVBLICA

Hadrian veiled standing l., sacrificing out of patera over altar

Inv. No. 122

$S / W / D \quad 17$ mm.; 3,4l gr.; $6 \mathrm{~h}$.

Ref. Cohen, 1481; BMCRE, 777; RIC, 290.

22. Obv. HADRIANVS AVG. COS. III. P. P.

Head of Hadrian, bare, $r$.

Rev. NILVS

Nilus reclining r.,holding cornucopiae and reed:

in front, hippotamus, below, crocodile.

Inv. No. 124

$S / W / D \quad 18 \mathrm{~mm}$.; 3,36 gr.; $6 \mathrm{~h}$.

Ref. $\quad$ Cohen, 989; BMCRE, 857; RIC, 310.

23. Obv. HADRIANVS AVG. COS. III. P. P.

Head of Hadrian, laureate, $r$.

Rev. ANNONA AVG.

Modius with corn-ears and popy.

Inv. No. 125

$S / W / D \quad 19 \mathrm{~mm}$.; 3.32 gr.; $6 \mathrm{~h}$.

Ref. Cohen, 170; BMCRE, 595; RIC, 230. 
Antoninus Pius (A.D. 138-161)
Mint of Rome (A.D. 138)

24. Obv. IMP. T. AEL. CAES. HADRI. ANTONINVS

Head of Antoninus Pius, bare, r.

Rev. $\quad$ AVG. PIVS. P. M. TR. P. COS. DES. II.

Minerva standing l., holding victory and resting 1 . hand on shield: against l.arm spear.

Inv. no. $\quad 136$

$S / W / D \quad 19$ mm.; 3.09 gr.; $6 \mathrm{~h}$.

Ref. Cohen, 67; BMCRE, 10; RIC, 9.

Mint of Rome (A.D. 140-143)

25. Obv. ANTONINVS AVG. PIVS P. P. Head of Antoninus Pius, bare, r.

Rev. TR. POT. COS. III.

She-wolf and twins $r$. in grotto.

Inv. no. $\quad 138$

$S / W / D \quad 17$ mm.; 3.14 gr.; $6 \mathrm{~h}$.

Ref. $\quad$ Cohen, 914; BMCRE, 232; RIC, 95.

26. Obv. ANTONINVS AVG. PIVS P. P. TR. P. COS. III.

Head of Antoninus Pius, laureate, $r$.

Rev. IMPERATOR II.

Victory standing 1 . holding wreath and palm.

Inv. No. 134

$S / W / D \quad 18 \mathrm{~mm} ; 3,33 \mathrm{gr} ; 7 \mathrm{~h}$.

Ref. Cohen, 437; BMCRE, 496; RIC, 111.

\section{Mint of Rome (A.D. 145-161)}

27. Obv. ANTONINVS AVG. PIVS P. P.

Head of Antoninus Pius, laureate, $r$.

Rev. $\quad$ COS IIII.

Winged thunderbolt on throne.

Inv. No. 139

$S / W / D \quad 17 \mathrm{~mm}$.; 3.58 gr.; $6 \mathrm{~h}$.

Ref. Cohen, 345; BMCRE, 537; RIC, 137.

28. $O b v$. ANTONINVS AVG. PIVS P. P. TR. P. XII.

Head of Antoninus Pius, laureate, $r$.

Rev COS IIII.

Fortuna standing l., holding rudder and cornucopiae.

Inv. No. 
$S / W / D \quad 19$ mm.; 3.32 gr.; $5 \mathrm{~h}$.

Ref. $\quad$ Cohen, 263; BMCRE, 668; RIC, 179.

\section{Mint of Rome (A.D. 150-151)}

29. Obv. ANTONINVS AVG. PIVS P. P. TR. P. XIIII.

Head of Antoninus Pius, laureate, $r$.

Rev. $\quad$ COS IIII

Felicitas standing l.; holding long caduceus and cornucopiae.

Inv. No. 132

$S / W / D \quad 19 \mathrm{~mm}$; $3.28 \mathrm{gr}$.; $6 \mathrm{~h}$.

Ref. Cohen, 254; BMCRE, 718; RIC, 193.

\section{Mint of Rome (A.D. 151-152)}

30. Obv. ANTONINVS AVG. PIVS P. P. TR. P. XV.

Head of Antoninus Pius, laureate, $r$.

Rev. [C] O [S] IIII

Vesta, standing l., holding simpulum and palladium.

Inv. No. 135

$S / W / D \quad 18 \mathrm{~mm} ; 3,37 \mathrm{gr} ; 6 \mathrm{~h}$.

Ref. Cohen, 196; BMCRE, 762; RIC, 203.

31.

$\begin{array}{ll} & \text { Similar } \\ \text { Inv. No. } & 133 \\ \text { S/W/D } & 19 \text { mm.; 3.38 gr.; 5h. } \\ \text { Ref. } & \text { Cohen, 196; BMCRE, 762; RIC, } 203 .\end{array}$

\section{Mint of Rome (A.D. 153-154)}

32. $O b v$. ANTONINVS AVG. PIVS P. P. TR. P. XVII.

Head of Antoninus Pius, laureate, $r$.

Rev. $\quad$ COS IIII.

Annona standing l., holding corn-ears and placing 1 . hand on modius set on ship, which is half seen.

Inv. No. 131

$S / W / D \quad 19$ mm.; 2.97 gr.; $7 \mathrm{~h}$.

Ref. Cohen, 291; BMCRE, 809; RIC, 231.

33. Obv. ANTONINVS AVG. PIVS P. P. TR. P. XVII.

Head of Antoninus Pius, laureate, $r$.

Rev. $\quad$ FORTVNA COS. IIII.

Fortuna standing r., holding rudder and cornucopiae.

Inv. No. $\quad 137$

$S / W / D \quad 17 \mathrm{~mm}$.; 3.11 gr.; $12 \mathrm{~h}$. 
Ref. $\quad$ Cohen, 271; BMCRE, ?; RIC, 232.

\section{Mint of Rome (A.D. 154-155)}

34. Obv. ANTONINVS AV[G. PIVS P. P. TR. P.] XVIII.

Head of Antoninus Pius, laureate, $r$.

Rev. $\quad$ COS

Vesta standing 1., sacrificing with patera over altar and holding palladium.

Inv. No. 128

$S / W / D \quad 18 \mathrm{~mm}$.; $3.07 \mathrm{gr}$.; $6 \mathrm{~h}$.

Ref. $\quad$ Cohen, 201; BMCRE, ; RIC, 238.

Mint of Rome (A.D. 159-160)

35. Obv. ANTONINVS AVG. PIVS P. P. TR. P. XXIII.

Head of Antoninus Pius, laureate, $r$.

Rev. $\quad$ PACI. AVG. COS. IIII

Pax standing l., holding branch and sceptre.

Inv. No. 130

$S / W / D \quad 18 \mathrm{~mm}$.; 3.32 gr.; $12 \mathrm{~h}$.

Ref. $\quad$ Cohen, 573; BMCRE, 981; RIC, 301.

36. $O b v$ ANTONINVS AVG. PIVS P. P. TR. P. XXIII.

Head of Antoninus Pius, laureate, $r$.

Rev. $\quad$ SALVTI AVG. COS. IIII.

Salus standing l., feeding snake coiled round altar and holding sceptre.

Inv. No. 126

$S / W / D \quad 17 \mathrm{~mm}$.; 3.32 gr.; $6 \mathrm{~h}$.

Ref. $\quad$ Cohen, 741; BMCRE, 988; RIC, 305.

37.

Similar

Inv. No. 127

$S / W / D \quad 18 \mathrm{~mm}$.; $3.18 \mathrm{gr}$.; $6 \mathrm{~h}$.

Ref. Cohen, 741; BMCRE, 988; RIC, 305.

Commemorative Coins struck after his death

Period of Marcus Aurelius

Mint of Rome (A.D. 176-180)

38. $O b v$. DIVUS ANTONINVS

Head of Antoninus Pius, bare, r.

Rev. CONSECRATIO

Pyre of four tiers, decorated with hangings and garlands, surmonted by quadriga. 
Inv. No. $\quad 140$

$S / W / D \quad 18 \mathrm{~mm} . ; 3.30$ gr.; $6 \mathrm{~h}$.

Ref. Cohen, 164; BMCRE, 57; RIC, 436.

\section{Faustina I (Wife of Antoninus Pius)}

Mint of Rome (A.D. 141)

39. $O b v$. DIVA FAVSTINA

Bust of Faustina, draped r.

Rev. [AVG]VSTA

Ceres, veiled, standing r., holding sceptre and corn-ears.

Inv. No. 146

$S / W / D \quad 17 \mathrm{~mm}$., 3.23 gr.; $6 \mathrm{~h}$.

Ref. $\quad$ Cohen, 93; BMCRE, 389; RIC, 358.

40. Obv. DIVA FAVSTINA

Bust of Faustina, draped $r$.

Rev. AVGVSTA

Ceres veiled, standing l., holding corn-ears and torch.

Inv. No. 144

$S / W / D \quad 18 \mathrm{~mm}$; 3.37 gr.; $7 \mathrm{~h}$.

Ref. Cohen, 78; BMCRE, 408; RIC, 360.

41. Obv. DIVA FAVSTINA

Bust of Faustina, draped $r$.

Rev. AVGVSTA

Vesta, veiled, seated 1., holding patera and sceptre.

Inv. No. $\quad 147$

$S / W / D \quad 19 \mathrm{~mm}$.; $3.19 \mathrm{gr}$; $12 \mathrm{~h}$.

Ref. Cohen, 119; BMCRE, 443; RIC, 371.

42. $O b v$. DIVA FAVSTINA

Bust of Faustina, draped r.

Rev. AVGVSTA

Pietas standing $\mathrm{l}$. by altar, raising $\mathrm{r}$.

hand and holding box of perfumes, with lid raised.

Inv. No. 145

$S / W / D \quad 19$ mm.; 2.95 gr.; $6 \mathrm{~h}$.

Ref. Cohen, 124; BMCRE, 449; RIC, 373.

Marcus Aurelius Caesar (A.D. 139-161)

Mint of Rome (A.D. 147-148)

43. Obv. AVRELIVS CAESAR AVG. P. II. F.

Bust of Marcus Aurelius, draped, head bare, $r$. 
Rev. TR. POT. II. COS. II.

Fides standing r., holding spear and resting 1 . hand on shield.

Inv. No. 156

$S / W / D \quad 18 \mathrm{~mm}$.; $3.06 \mathrm{gr}$; $6 \mathrm{~h}$.

Ref. $\quad$ Cohen, 613; BMCRE, 645; RIC, 440a.

Mint of Rome (A.D. 159-160)

44. Obv. AVRELIVS CAES AVG. P. II. F.

Head of Marcus Aurelius, bare, r.

Rev. TR. POT. XIIII. COS. II.

Minerva, helmeted, advancing r.,brandishing javelin and holding shield.

Inv. No. 153

$S / W / D \quad 18 \mathrm{~mm}$.; 3.23 gr.; $8 \mathrm{~h}$.

Ref. $\quad$ Cohen, 762, BMCRE, 636; RIC, 483.

Marcus Aurelius Augustus (A.D. 161-180)

Mint of Rome (A.D. 161)

45. Obv. IMP. M. AVREL. ANTONINVS AVG.

Head of Marcus Aurelius, bare, r.

Rev. $\quad$ PROV. DEOR. TR. P. XV. COS. III.

Providentia standing l.,holding globe and cornucopiae.

Inv. No. 151

$S / W / D \quad 17 \mathrm{~mm} . ; 3.28$ gr.; $12 \mathrm{~h}$.

Ref. $\quad$ Cohen, 507; BMCRE, 15; RIC, 22.

Mint of Rome (A.D. 161-162)

46. Obv. IMP. M. AVREL. ANTONINVS AVG.

Head of Marcus Aurelius, bare, $r$.

Rev. CONCORD. AVG. TR. P. XVI. COS. III.

Concordia seated l., holding patera and resting 1 . arm on statue of Spes; cornucopiae under seat.

Inv. No. 158

$S / W / D \quad 18 \mathrm{~mm} . ; 3.06$ gr.; $12 \mathrm{~h}$.

Ref. $\quad$ Cohen, 35; BMCRE, 177; RIC, 35.

Mint of Rome (A.D. 162-163)

47. Obv. IMP M. ANTONINVS AVG

Head of Marcus Aurelius, bare, $r$.

Rev. PROV. DEOR. TR. P. XVII. COS. III.

Providentia standing 1 ., holding globe and cornucopiae.

Inv. No. 161 
$S / W / D \quad 17$ mm.; 3.18 gr.; $12 \mathrm{~h}$.

Ref. $\quad$ Cohen, 526; BMCRE, 218; RIC, 70.

\section{Mint of Rome (A.D. 163-164)}

48. $O b v$. ANTONINVS AVG. ARMENIACVS

Head of Marcus Aurelius, laureate, $r$.

Rev. P. M.TR. P. XVIII. IMP. II. COS. III.

Inv. No. 157

$S / W / D \quad 16 \mathrm{~mm}$.; 3.36 gr.; $6 \mathrm{~h}$.

Ref. $\quad$ Cohen, 469; BMCRE, 264; RIC, 92.

Mint of Rome (A.D. 169-170)

49. Obv. M. ANTONINVS AVG. TR. P. XXIIII.

Head of Marcus Aurelius, laureate, $r$.

Rev. FELICITAS AVG. COS. III.

Felicitas standing l., holding caduceus and sceptre.

Inv. No. 150

$S / W / D \quad 18 \mathrm{~mm}$.; 3.34 gr.; $10 \mathrm{~h}$.

Ref. Cohen, 181; BMCRE, 517; RIC, 218.

50. Obv. M. ANTONINVS AVG. TR. P. XXIIII.

Head of Marcus Aurelius, laureate, $r$.

Rev. SALVTI AVG. COS. III.

Salus standing l., holding sceptre and feeding snake twined round altar.

Inv. No. 148

$S / W / D \quad 19$ mm.; 3.44 gr.; $7 \mathrm{~h}$.

Ref. Cohen, 546; BMCRE, 525; RIC, 222.

Mint of Rome (A.D. 176-177)

51. Obv. M. ANTONINVS AVG. GERM. SARM.

Head of Marcus Aurelius, laureate, $r$.

Rev. TR. P. XXX. IMP. VIII. COS. III.

Aequitas standing l., holding scales and cornucopiae.

Inv. No. $\quad 152$

$S / W / D \quad 19 \mathrm{~mm}$; $3.40 \mathrm{gr} ; 7 \mathrm{~h}$.

Ref. $\quad$ Cohen, ?; BMCRE, 672; RIC, 359 (similar).

52. Obv. M.ANTONINVS AVG. GERM. SARM.

Head of Marcus Aurelius, laureate, $r$.

Rev. TR. P. XXXI. IMP. VIII. COS. III. P. P.

Salus standing l., holding sceptre and feeding snake twined round altar. 
Inv. No. $\quad 149$

$S / W / D \quad 21 \mathrm{~mm} . ; 3.28$ gr.; $11 \mathrm{~h}$.

Ref. $\quad$ Cohen, 944; BMCRE, 733; RIC, 373.

Mint of Rome (A.D. 177-178)

53. Obv. M. AVREL. ANTONINVS AVG.

Head of Marcus Aurelius, laureate, r.

Rev. TR. P. XXXII. IMP.VIIII. COS. III. P. P.

Minerva standing l., holding olive-branch and leaning on shield; spear resting on 1 . arm.

Inv. No. 154

$S / W / D \quad 18 \mathrm{~mm}$.; $3.32 \mathrm{gr}$.; $5 \mathrm{~h}$.

Ref. $\quad$ Cohen, 956; BMCRE, ?; RIC, 387.

\section{Faustina II (A.D. 161-180)}

Mint of Rome (A.D. 161)

54. Obv. FAVSTINAE AVG. PII. AVG. FIL.

Bust of Faustina II,draped, band of pearls, r.

Rev. VENVS

Venus, standing l., holding apple and round rudder dolphin twined.

Inv. No. 141

$S / W / D \quad 19$ mm.; 3.41 gr.; $6 \mathrm{~h}$.

Ref. $\quad$ Cohen, 267; BMCRE, 1067; RIC, 517a.

55.

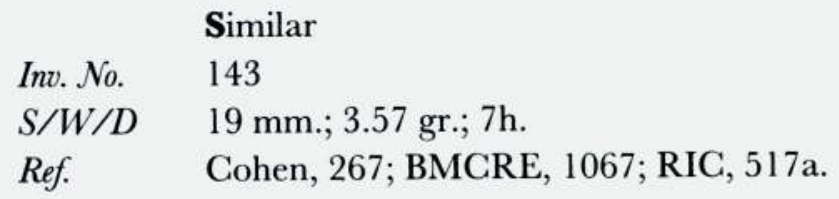

56. Obv. FAVSTINAE AVG. PII. AVG. FIL.

Bust of Faustina II, draped, band of pearls, r.

Rev. VENVS

Venüs standing l., holding apple and rudder.

Inv. No. 142

$S / W / D \quad 19$ mm.; 3.50 gr.; 7h.

Ref. Cohen, ?; BMCRE, ?; RIC, 516.

Mint of Rome (A.D. 179)

57. Obv. FAVSTINA AVGVSTA

Bust of Faustina II, draped, band of pearls, r.

Rev. FECVNDITAS

Fecunditas, standing r., holding sceptre and infant. 


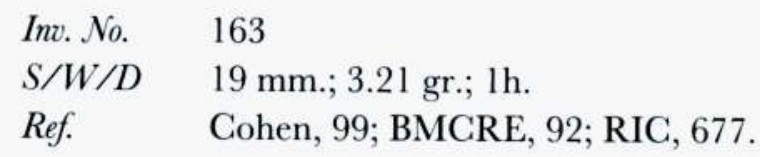

58. Obv. FAVSTINA AVGVSTA

Bust of Faustina II, draped, band of pearls, $r$.

Rev. HILARITAS

Inv. No. 162

$S / W / D \quad 19$ mm.; 3.16 gr.; $5 \mathrm{~h}$.

Ref. Cohen, 111; BMCRE, 100; RIC, 686.

59. Obv. FAVSTINA AVGVSTA

Bust of Faustina II, draped, band of pearls, r.

Rev. SAECVLI FELICIT.

Pulvinar on which are Commodus and Antoninus.

Inv. No. 164

$S / W / D \quad 18 \mathrm{~mm}$.; $3.41 \mathrm{gr}$.; $5 \mathrm{~h}$.

Ref. Cohen, 191; BMCRE, 136; RIC, 712.

60.

Similar

$S / W / D \quad 18 \mathrm{~mm} . ; 3.39 \mathrm{gr} ; 6 \mathrm{~h}$.

Ref. Cohen, 191; BMCRE, 136; RIC, 712.

\section{Lucius Verus (A.D. 161-169)}

Mint of Rome (A.D. 163-164)

61. Obv. L. VERVS AVG. ARMENIACVS

Head of Lucius Verus, bare, $r$.

Rev. TR.P. IIII. IMP. II. COS.II. ARMEN.

Armenia seated l., surrounded by arms, supporting chin with r.hand; before her standart.

Inv. No. 168

$S / W / D \quad 17$ mm.; 3.17 gr.; $6 \mathrm{~h}$.

Ref. Cohen, 8; BMCRE, 298; RIC, 505 (similar).

62. Obv. L. VERVS AVG. ARMENIACVS

Head of Lucius Verus, laureate, $r$.

Rev. TR. P. IIII. IMP. II. COS. II.

Inv. No. 167

$S / W / D \quad 19$ mm.; 3.22 gr.; $6 \mathrm{~h}$.

Ref. Cohen, 230; BMCRE, 287; RIC, 516. 


\section{Mint of Rome (A.D. 166)}

63. Obv. L. VERVS AVG. ARM. PARTH. MAX.

Head of Lucius Verus, laureate, $r$.

Rev. TR. P. VI. IMP. IIII.COS. II.

Victory, half-draped, standing r.;

holds palm and places on a palm-tree ashield inscribed VIC PAR.

Inv. No. 155

$S / W / D \quad 16 \mathrm{~mm}$.; $3.51 \mathrm{gr}$.; $6 \mathrm{~h}$.

Ref. Cohen, 279; BMCRE, 432; RIC, 566.

\section{Mint of Rome (A.D. 166-167)}

64. Obv. L. VERVS AVG. ARM. PARTH. MAX.

Head of Lucius Verus, laureate, $r$.

Rev. TR. P. VII. IMP. IIII. COS. III.

Victory walking 1 , holding wreath and palm.

Inv. No. 166

$S / W / D \quad 21 \mathrm{~mm}$.; 3.25 gr.; $6 \mathrm{~h}$.

Ref. Cohen, 295; BMCRE, 450; RIC, 574.

Commodus (A.D. 177-192)

Mint of Rome (A.D. 180)

65. Obv. M. COMMODVS ANTONINVS AVG.

Bust of Commodus, laureate r., with drapery on 1 .

shoulder.

Rev. TR. P. V. IMP. IIII. COS. II. P. P.

Fortuna seated 1., holding rudder and cornucopiae.

Inv. No. 175

$S / W / D \quad 19 \mathrm{~mm}$.; 3.00 gr.; $6 \mathrm{~h}$.

Ref. $\quad$ Cohen, 786; BMCRE, 7; RIC, 6b.

66. $O b v$ DIVVS M. ANTONINVS PIVS

Head of Marcus Aurelius, bare, $r$.

Rev. CONSECRATIO

Eagle standing r. on thunderbolt, head $\mathrm{r}$.

Inv. No. $\quad 159$

$S / W / D \quad 18 \mathrm{~mm}$.; $2.50 \mathrm{gr}$.; $6 \mathrm{~h}$.

Ref. Cohen, 83; BMCRE, 22; RIC, 269.

67. Obv. DIVVS M. ANTONINVS PIVS

Head of Marcus Aurelius, bare, r.

Rev. CONSECRATIO 
Funeral pyre, in four storeys, garlanded and adorned with statues: on top, Marcus Aurelius in quadriga.

Inv. No. 160

$S / W / D \quad 17 \mathrm{~mm}$.; 2.89 gr.; $12 \mathrm{~h}$.

Ref. $\quad$ Cohen, 97; BMCRE, 27; RIC, 275.

\section{Mint of Rome (A.D. 181)}

68. Obv. M. COMMODVS ANTONINVS AVG.

Head of Commodus, laureate, $r$.

Rev. LIB. AVG. IIII. TR. P. VI. IMP. IIII. COS. III. P. P.

Inv. No. $\quad 179$

$S / W / D \quad 18 \mathrm{~mm}$.; 2.55 gr.; $6 \mathrm{~h}$.

Ref. Cohen, 307; BMCRE, 53; RIC, 22.

Mint of Rome (A.D. 183)

69. Obv. M. COMMODVS ANTON. AVG. PIVS

Head of Commodus, laureate, $r$.

Rev. TR. P. VIII. IMP. VI. COS. IIII. P. P.

Mars advancing r., holding spear and trophy.

Inv. No. 172

$S / W / D \quad 17 \mathrm{~mm}$; $2.69 \mathrm{gr}$; $12 \mathrm{~h}$.

Ref. Cohen, 878; BMCRE, 102; RIC, 54.

Mint of Rome (A.D. 183-184)

70. Obv. M. COMMODVS ANTON. AVG. PIVS.

Head of Commodus, laureate, $r$.

Rev. TR. P. VIIII. IMP. VI. COS. IIII. P. P.

Minerva advancing $\mathrm{r}$., brandishing javelin and holding shield: at feet, r., owl.

Inv. No. 180

$S / W / D \quad 18 \mathrm{~mm}$.; $3.13 \mathrm{gr}$; $6 \mathrm{~h}$.

Ref. Cohen, 424 (similar); BMCRE, 131; RIC, 72.

Mint of Rome (A.D. 184)

71. Obv. M. COMM. ANTON. AVG. PIVS. BRIT.

Head of Commodus, laureate, $r$.

Rev. P. M. TR. P. VIIII. IMP. VII. COS. IIII. P. P.

Modius and corn-ears.

Inv. No. 185

$S / W / D \quad 17$ mm.; 3.29 gr.; $6 \mathrm{~h}$. 
Ref. $\quad$ Cohen, 467; BMCRE, 143; RIC, 94.

\section{Mint of Rome (A.D. 185)}

72. Obv. M. COMM. ANT. P. FEL. AVG. BRIT.

Head of Commodus, laureate, $r$.

Rev. $\quad$ P. M. TR. P. XI. IMP. VII. COS. V. P. P.

Jupiter seated l., holding thunderbolt and sceptre.

Inv. No. 177

$S / W / D \quad 18 \mathrm{~mm}$.; 2.90 gr.; $6 \mathrm{~h}$.

Ref. Cohen, 486; BMCRE, 183; RIC, 117.

73. Obv. M. COMM. ANT. P. FEL. AVG. BRIT.

Head of Commodus, laureate, $r$.

Rev. $\quad$ P. M. TR. P. XI. IMP. VII. COS. V. P. P.

Victory advancing l, holding wreath and palm.

Inv. No. $\quad 190$

$S / W / D \quad 17 \mathrm{~mm} . ; 2.91$ gr.; $11 \mathrm{~h}$.

Ref. $\quad$ Cohen, 492; BMCRE, 190; RIC, 122a.

74. Obv. M. COMM. ANT. P. FEL. AVG. BRIT.

Head of Commodus, laureate, $r$.

Rev. P. M. TR. P. XI. IMP. VII. COS. V. P. P.

Commodus seated 1., on curule chair, holding globe, crowned by flying Victory.

Inv. No. $\quad 186$

$S / W / D . \quad 18 \mathrm{~mm}$.; $2.57 \mathrm{gr}$; $5 \mathrm{~h}$.

Ref. $\quad$ Cohen, 505; BMCRE, 193; RIC, 125.

75. Obv. M. COMM. ANT. P. FEL. AVG. BRIT.

Head of Commodus, laureate, $r$.

Rev. $\quad$ FID. EXERC. P. M. TR. P. XI. IMP. VII. COS. V. P. P.

Commodus standing 1 , , on platform, holding sceptre and haranguing three soldiers.

Inv. No. 182

$S / W / D . \quad 17 \mathrm{~mm} . ; 2.67$ gr.; $11 \mathrm{~h}$.

Ref. Cohen, 143; BMCRE, 199; RIC, 130.

Mint of Rome (A.D.186-187)

76. $O b v$ M.COMM. ANT. P. FEL. AVG. BRIT

Head of Commodus, laureate, $r$.

Rev. HILAR. AVG. P.M. TR. P. XII. IMP. VIII. COS. V. P.P.

Hilaritas standing 1 ., holding branch and long palm. 
Inv. No. 188

$S / W / D . \quad 17 \mathrm{~mm} ; 2.26 \mathrm{gr} ; 12 \mathrm{~h}$.

Ref. $\quad$ Cohen, 212;BMCRE, 210;RIC, 150a.

77. Obv. M. COMM. ANT. P. FEL. AVG. BRIT,

Head of Commodus, laureate, $r$.

Rev. VOT. SOL. DEC. P. M. TR.P. XII. IMP. VIII. COS. V.

P. P.

Commodus standing l, sacrificing over tripod beside which is a victim.

Inv. No. $\quad 178$

$S / W / D . \quad 19 \mathrm{~mm} . ; 2.92 \mathrm{gr}$; $11 \mathrm{~h}$.

Ref. Cohen, 1001; BMCRE, 229; RIC, 161.

\section{Mint of Rome (A.D.187-188)}

78. Obv. M. COMM. ANT. P. FEL. AVG. BRIT.

Head of Commodus, laureate, $r$.

Rev. P. M. TR. P. XIII. IMP. VIII. COS. V. P. P.

Aequitas standing l., holding scales and cornucopiae.

Inv. No. 183

$S / W / D \quad 18 \mathrm{~mm}$.; 3.33 gr.; $11 \mathrm{~h}$.

Ref. Cohen, 537; BMCRE, 242; RIC, 164.

79. Obv. M. COMM. ANT. P. FEL. AVG. BRIT.

Head of Commodus, laureate, $r$.

Rev. P. M.TR. P. XIII. IMP. VIII. COS. V. P. P.

Genius standing l.,holding patera and corn-ears.

Inv. No. 195

$S / W / D \quad 17$ mm.; 3.04 gr.; $12 \mathrm{~h}$.

Ref. Cohen, 532; BMCRE, 245; RIC, 167.

80. Obv. M. COMM. ANT. P. FEL. AVG. BRIT.

Head of Commodus, laureate, $r$.

Rev. P.M.TR. P. XIII. IMP. VIII.COS. V. P. P.

Libertas standing front, head l., holding pileus and rod transverse above head.

Inv. No 192

$S / W / D \quad 18 \mathrm{~mm}$.; $2.81 \mathrm{gr}$; $12 \mathrm{~h}$.

Ref. $\quad$ Cohen, 542; BMCRE, 248; RIC, 168.

81.

$\begin{array}{ll} & \text { Similar } \\ \text { Inv. No. } & 187 \\ \text { S/W/D } & 18 \mathrm{~mm} . ; 2.78 \mathrm{gr} . ; 12 \mathrm{~h} . \\ \text { Ref. } & \text { Cohen, 542; BMCRE, 248; RIC, } 168 .\end{array}$


82.

\begin{tabular}{ll}
\multicolumn{1}{c}{ Similar } \\
Inv. No. & 184 \\
S/W/D & $17 \mathrm{~mm} . ; 3.13 \mathrm{gr} . ; 6 \mathrm{~h}$. \\
Ref. & Cohen, 542; BMCRE, 248; RIC, 168.
\end{tabular}

\section{Mint of Rome (A.D. 188-189)}

83. $O b v . \quad$ M. COMM. ANT. P. FEL. AVG. BRIT.

Head of Commodus, laureate, $r$.

Rev. MART. PAC. P. M. TR. P. XIIII.COS. V. P. P.

Mars standing l., holding branch and spear: at feet, arms.

Inv. No. 181

$S / W / D \quad 18 \mathrm{~mm}$.; 3.54 gr.; $6 \mathrm{~h}$.

Ref. Cohen, 350; BMCRE, 256; RIC, 175.

\section{Mint of Rome (A.D. 186-189)}

84. Obv. M.COMM. ANT. P. FEL. AVG. BRIT.

Head of Commodus, laureate, $r$.

Rev. FORTVNAE MANENTI C. V. P. P.

Fortuna seated l., holding horse by bridle and cornicopiae.

Inv. No. 196

$S / W / D \quad 16 \mathrm{~mm} . ; 2.68$ gr.; $6 \mathrm{~h}$.

Ref. Cohen, 168; BMCRE, 231; RIC, 191a.

85. Obv. M. COMM. ANT. P. FEL. AVG. BRIT.

Head of Commodus, laureate, $r$.

Rev. OPTIME MAXIME C. V. P. P.

Jupiter standing l., holding thunderbolt and sceptre.

Inv. No. 194

$S / W / D \quad 18 \mathrm{~mm}$.; $3.53 \mathrm{gr}$.; $6 \mathrm{~h}$.

Ref. $\quad$ Cohen, 387; BMCRE, 233; RIC, 192.

$86 . \quad$ Similar

Inv. No. $\quad 169$

$S / W / D . \quad 18 \mathrm{~mm} . ; 2.65$ gr.; $12 \mathrm{~h}$. .

Ref. $\quad$ Cohen, 387; BMCRE, 233; RIC, 192.

\section{Mint of Rome (A.D. 189)}

87. Obv. M. COMM. ANT. P. FEL. AVG. BRIT. P. P.

Head of Commodus, laureate, $r$.

Rev. LIBERALITAS AVG. VII.

Liberalitas standing 1., holding abacus and cornucopiae.

Inv. No. 193 
$S / W / D . \quad 19 \mathrm{~mm} . ; 2.87$ gr.; $12 \mathrm{~h}$.

Ref. Cohen, 323; BMCRE, 286; RIC, 202a.

\section{Mint of Rome (A.D. 190-191)}

88. Obv. M. COMM. ANT. P. FEL. AVG. BRIT. P. P. Head of Commodus, laureate, $r$.

Rev. $\quad$ APOL. PAL. P. M. TR. P. XVI. COS. VI.

Apollo standing front, head r., holding plectrum and lyre resting on column.

Inv. No 174

$S / W / D . \quad 18 \mathrm{~mm}$.; 3.05 gr.; $12 \mathrm{~h} .$.

Ref. Cohen, 24; BMCRE, 292; RIC, 218.

89. Obv. M. COMM. ANT. P. FEL. AVG. BRIT. P. P.

Head of Commodus, laureate, $r$.

Rev. FIDEI. COH. P. M. TR. P. XVI. COS. VI.

Fides standing l., holding corn-ears and standart.

Inv. No 189

$S / W / D . \quad 17 \mathrm{~mm}$.; $2.50 \mathrm{gr}$; $1 \mathrm{~h}$.

Ref. $\quad$ Cohen, 127; BMCRE, 298; RIC, 220.

90. Obv. M. COMM. ANT. P. FEL. AVG. BRIT. P. P.

Head of Commodus, laureate, $r$.

Rev. MIN. AVG. P. M.TR. P. XVI. COS. VI.

Minerva hurrying r., head turned back l., holding branch and spear and shield.

Inv. No 171

$S / W / D . \quad 17 \mathrm{~mm} . ; 2.86$ gr.; $7 \mathrm{~h}$.

Ref. Cohen, 358; BMCRE, 302; RIC, 222a.

Mint of Rome (A.D. 192)

91. Obv. L. AEL. AVREL. COMM. AVG. P. FEL.

Head of Commodus, laureate, $r$.

Rev. P. M. TR. P. XVII. IMP. VIII. COS. VII. P. P.

Fides standing l., holding sceptre and cornucopiae: star in field.

Inv. No 173

$S / W / D . \quad 18 \mathrm{~mm} . ; 3.00 \mathrm{gr}$; $11 \mathrm{~h}$.

Ref. Cohen, 586; BMCRE, 317; RIC, 234.

92.

$\begin{array}{ll}\text { Snv. No } & \text { Similar } \\ 191\end{array}$


$S / W / D . \quad 17 \mathrm{~mm} . ; 2.55$ gr.; $6 \mathrm{~h}$.

Ref. Cohen, 586; BMCRE, 319; RIC, 234.

93. Obv. L. AEL. AVREL. COMM. AVG. P. FEL.

Head of Commodus, laureate, $r$.

Rev. PM. TR. P. XVII. IMP. VIII. COS. VII. P. P.

Victory advancing l., holding wreat and palm: sometimes, star in field.

Inv. No $\quad 176$

$S / W / D . \quad 19 \mathrm{~mm}$; $3.00 \mathrm{gr} . ; 6 \mathrm{~h}$.

Ref. Cohen, 568; BMCRE, 329; RIC, 237.

94.

\begin{tabular}{ll}
\multicolumn{2}{c}{ Similar } \\
Inv. No & 170 \\
S/W/D. & $18 \mathrm{~mm}$; 3.46 gr.; 12h. \\
Ref. & Cohen, 568; BMCRE, 329; RIC, 237.
\end{tabular}

95. Obv. L. AEL. AVREL. COMM. AVG. P. FEL.

Head of Commodus, laureate, $r$.

Rev. HERCVLI ROMANO AVG.

Herkül standing l., holding club and lion-skin in l., and with r. crowning trophy.

Inv. No 197

$S / W / D . \quad 18 \mathrm{~mm} . ; 2.66$ gr.; $12 \mathrm{~h}$.

Ref. Cohen,?; BMCRE, 346 A; RIC, 254a.

Crispina (Wife of Commodus)

Mint of Rome (A.D. 180)

96. Obv. CRISPINA AVGVSTA

Bust of Crispina, draped $r$.

Rev. CERES

Ceres standing $1 .$, holding corn-ears and torch.

Inv. No 198

$S / W / D . \quad 17 \mathrm{~mm}$.; $3.17 \mathrm{gr}$.; $6 \mathrm{~h}$.

Ref. Cohen, 1; BMCRE, 33; RIC, 276.

Clodius Albinus (A.D. 195-197)

Mint of Rome (A.D. 193)

97. Obv. D. CLOD. SEPT. ALBIN. CAES

Head of Albinus, bare, $r$.

Rev. FELICITAS COS II.

Felicitas standing 1., holding caduceus and sceptre.

Inv. No 200 
$S / W / D . \quad 17 \mathrm{~mm} . ; 3.39$ gr.; $12 \mathrm{~h}$.

Ref. Cohen, 15; BMCRE, 91; RIC, 4.

\section{Mint of Rome (A.D. 193-194)}

98. Obv. D. CLOD. SEPT. ALBIN. CAES.

Head of Albinus, bare, $r$.

Rev. ROMAE AETERNAE

Roma, helmeted, seated l.on shield, holding palladium and sceptre.

Inv. No 199

$S / W / D . \quad 17 \mathrm{~mm}$; $2.79 \mathrm{gr} ; 6 \mathrm{~h}$.

Ref. Cohen, 61; BMCRE, 45; RIC, 11.

Septimus Severus (A.D. 193-211)

Mint of Rome (A.D. 193-194)

99. Obv. IMP. CAE. L. SEP. SEV. PERT. AVG.

Head of Septimus Severus, laureate, $r$.

Rev. LIBERAL. AVG. COS.

Liberalitas standing l., holding account-board and cornucopiae.

Inv. No. 250

$S / W / D . \quad 17$ mm.; 3.11 gr.; $5 \mathrm{~h}$.

Ref. $\quad$ Cohen, 281; BMCRE, 2; RIC, 18.

100.

Similar

Inv. No. 247

$S / W / D . \quad 18 \mathrm{~mm}$.; $2.49 \mathrm{gr}$; $7 \mathrm{~h}$.

Ref. $\quad$ Cohen, 281; BMCRE, 2; RIC, 18.

101.

$\begin{array}{ll} & \text { Similar } \\ \text { Inv. No. } & 243 \\ \text { S/W/D. } & 17 \mathrm{~mm} . ; 2.86 \text { gr.; 6h. } \\ \text { Ref. } & \text { Cohen, 281; BMCRE, 2; RIC, } 18 .\end{array}$

102.

Similar

Inv. No. 239

$S / W / D . \quad 17 \mathrm{~mm}$.; $3.08 \mathrm{gr}$.; $6 \mathrm{~h}$.

Ref. Cohen, 281; BMCRE, 2; RIC, 18.

103. Obv. IMP. CAE. L. SEP. SEV. PERT. AVG.

Head of Septimus Severus, laureate, $r$.

Rev. VIRT. AVG. TR. P. COS.

Virtus, helmeted, standing l., holding Victory and spear reversed.

Inv. No. 251 
$S / W / D . \quad 16 \mathrm{~mm}$.; $2.71 \mathrm{gr}$.; $6 \mathrm{~h}$.

Ref. $\quad$ Cohen, 752; BMCRE, 33; RIC, 24.

$104 . \quad$ Similar

Inv. No. 221

$S / W / D . \quad 18 \mathrm{~mm} . ; 2.80 \mathrm{gr}$.; $6 \mathrm{~h}$.

Ref. Cohen, 752; BMCRE, 33; RIC, 24.

Mint of Rome (A.D. 194-195)

105. Obv. L. SEPT. SEV. PERT. AVG. IMP. IIII. Head of Septimus Severus, laureate, $r$.

Rev. APOLLINI AVGUSTO

Apollon standing l., holding patera and lyre.

Inv. No. 224

$S / W / D . \quad 18 \mathrm{~mm}$.; 2.95 gr.; $6 \mathrm{~h}$.

Ref. Cohen, 42; BMCRE, 78; RIC, 40.

\section{Mint of Rome (A.D. 195-196)}

106. Obv. L. SEPT. SEV. PERT. AVG. IMP. VII.

Head of Septimus Severus, laureate, $r$.

Rev. P. M. TR. P. III. COS. II. P. P.

Mars advancing l., holding spear and trophy.

Inv. No. 211

$S / W / D \quad 18 \mathrm{~mm}$; $3.10 \mathrm{gr}$; $12 \mathrm{~h}$.

Ref. Cohen, 397; BMCRE, 122; RIC, 67.

\section{Mint of Rome (A.D. 196-197)}

107. Obv. L. SEPT. SEV. PERT. AVG. IMP. VIII.

Head of Septimus Severus, laureate, $r$.

Rev. ARAB. ADIAB. COS. II. P. P.

Victory advancing l., holding wreath and trophy.

Inv. No. 249

$S / W / D \quad 16 \mathrm{~mm} . ; 3.14$ gr.; $12 \mathrm{~h}$.

Ref. $\quad$ Cohen, 51; BMCRE, 157; RIC, 76.

108. Obv. L. SEPT. SEV. PERT. AVG. IMP. VIII.

Head of Septimus Severus, laureate, r.

Rev. HERCVLI DEFENS

Hercules standing r., resting on club and holding bow and lion-skin on l.arm.

Inv. No. 242

$S / W / D \quad 17$ mm.;3.12 gr.; $5 \mathrm{~h}$. 
Ref. Cohen, 210; BMCRE,?; RIC, 79.

109. Obv. L. SEPT. SEV. PERT. AVG. IMP. VIII.

Head of Septimus Severus, laureate, $r$.

Rev. $\quad$ P. M. TR. P. IIII. COS. II. P. P.

Pax seated l., holding branch and sceptre.

Inv. No. 231

$S / W / D \quad 19 \mathrm{~mm} . ; 3.26$ gr.; $12 \mathrm{~h}$.

Ref. Cohen, 429; BMCRE, 142; RIC, 85.

Mint of Rome (A.D. 197)

110. Obv. L. SEPT. SEV. PERT. AVG. IMP. VIIII.

Head of Septimus Severus, laureate, $r$.

Rev. HERCVLI DEFENS

Hercules standing r., resting on club and holding bow and lion-skin on 1., arm.

Inv. No. 237

$S / W / D \quad 15 \mathrm{~mm}$.; $2.78 \mathrm{gr}$.; $6 \mathrm{~h}$.

Ref. Cohen, 211; BMCRE, 218; RIC, 97.

111. Similar

Inv. No. 248

$S / W / D \quad 16 \mathrm{~mm} . ; 3.26 \mathrm{gr}$; $6 \mathrm{~h}$.

Ref. Cohen, 211; BMCRE, 218; RIC, 97.

112. Obv. I. SEPT. SEV. PERT. AVG. IMP. VIIII.

Head of Septimus Severus, laureate, $r$.

Rev. $\quad$ LIBERO PATRI

Bacchus(Liber) standing front, head 1., r. hand on head (crowning himself?) holding thyrsus in 1.hand: at feet l., leopard.

Inv. No. 244

$S / W / D \quad 18 \mathrm{~mm}$; $3.22 \mathrm{gr}$; $12 \mathrm{~h}$.

Ref. $\quad$ Cohen, 304; BMCRE, 222; RIC, 99.

113. Obv. L. SEPT. SEV. PERT. AVG. IMP. VIIII.

Head of Septimus Severus, laureate, $r$.

Rev. $\quad$ P. M. T.R. P. V. COS. II. P. P.

Sol, radiate, standing 1 ., raising $\mathrm{r}$. hand and holding whip in $\mathrm{l}$.

Inv. No. 245

$S / W / D \quad 17$ mm.; 3.23 gr.; $12 \mathrm{~h}$.

Ref. Cohen, 433; BMCRE, 227; RIC, 101. 
114. Similar

Inv. No. 209

$S / W / D \quad 20 \mathrm{~mm}$.; 2.72 gr.; $12 \mathrm{~h}$.

Ref. Cohen, 433; BMCRE, 227; RIC, 101.

115. Obv. L. SEPT. SEV. PERT. AVG. IMP. VIIII.

Head of Septimus Severus, laureate, $r$.

Rev. $\quad$ P. M. T. R. P. V. COS. II. P. P.

Fortuna standing $\mathrm{l}$, holding rudder on globe and cornucopiae.

Inv. No. 252

$S / W / D \quad 15 \mathrm{~mm}$.; 3.33 gr.; $5 \mathrm{~h}$.

Ref. Cohen, 442; BMCRE, 229; RIC, 104.

Mint of Rome (A.D. 197-198)

116. $O b v$ L. SEPT. SEV. PERT. AVG. IMP. X.

Head of Septimus Severus, laureate, $r$.

Rev. ANNONAE AVGG.

Annona standing l., foot on prow, holding corn-ears and cornucopiae.

Inv. No. 204

$S / W / D \quad 18 \mathrm{~mm}$.; $3.26 \mathrm{gr}$.; lh.

Ref. $\quad$ Cohen, 37; BMCRE, 239; RIC, 107.

117. $\mathrm{Obv}$.

L. SEPT. SEV. PERT. AVG. IMP. X.

Head of Septimus Severus, laureate, $r$.

Rev. $\quad$ MARTI VICTORI

Mars standing r., holding shield and spear reversed.

Inv. No. 236

$S / W / D \quad 18 \mathrm{~mm} . ; 3.35$ gr.; $12 \mathrm{~h}$.

Ref. Cohen, 319; BMCRE,?; RIC, 114.

118. Obv. L. SEPT. SEV. PERT. AVG. IMP. X.

Head of Septimus Severus, laureate, $r$.

Rev. VICT. AVGG. COS. II. P. P.

Victory advancing 1 , holding wreath and palm.

Inv. No. 232

$S / W / D \quad 17 \mathrm{~mm}$.; 3.52 gr.; $6 \mathrm{~h}$.

Ref. $\quad$ Cohen, 694; BMCRE, 258; RIC, 120c.

\section{Mint of Rome (A.D. 198-200)}

119. Obv. L. SEPT. SEV. AVG. IMP. XI. PART. MAX.

Head of Septimus Severus, laureate, $r$.

Rev. $\quad$ COS. II. P. P. 
Victory advancing 1 , holding wreath and palm.

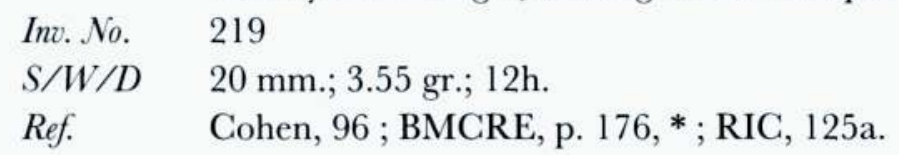

120. $O b v$.

Rev. Head of Septimus Severus,

Victory flying l., holding wreath in both hands: before her, shield on low base.

Inv. No. 216

$S / W / D \quad 18 \mathrm{~mm}$; $3.19 \mathrm{gr}$; $6 \mathrm{~h}$.

Ref. Cohen, 719; BMCRE, 139; RIC, 144b.

121. Similar

Inv. No. 222

$S / W / D \quad 18 \mathrm{~mm} ; 3.55 \mathrm{gr} . ; 7 \mathrm{~h}$.

Ref. $\quad$ Cohen, 719;BMCRE, 139 ;RIC, 144b.

Mint of Rome (A.D. 200-201)

122. Obv. SEVERVS AVG. PART. MAX.

Head of Septimus Severus, laureate, r.

Rev. PROVID. AVGG.

Providentia standing l., holding wand over globe and sceptre.

Inv. No. 207

$S / W / D \quad 18 \mathrm{~mm}$.; 3.38 gr.; $12 \mathrm{~h}$.

Ref. Cohen, 586; BMCRE, 197; RIC, 166.

123. Obv. SEVERVS AVG. PART. MAX.

Head of Septimus Severus, laureate, $r$.

Rev. RESTITVTOR VRBIS

Septimus Severus standing l., sacrificing with patera over tripod and holding spear.

Inv. No. 228

$S / W / D \quad 18 \mathrm{~mm}$; $3.09 \mathrm{gr}$; $7 \mathrm{~h}$.

Ref. $\quad$ Cohen, 599; BMCRE, 202; RIC, 167a.

124. Obv. SEVERVS AVG. PART. MAX.

Head of Septimus Severus, laureate, $r$.

Rev. VIRT. AVGG.

Virtus standing 1., holding Victory and resting on shield, spear against 1., arm.

Inv. No. 203 
$S / W / D \quad 18 \mathrm{~mm}$.; 3.16 gr.; $6 \mathrm{~h}$.

Ref. Cohen, 761; BMCRE, 211; RIC, $171 \mathrm{a}$.

125.

Similar

Inv. No. 206

$S / W / D \quad 19 \mathrm{~mm}$; $3.30 \mathrm{gr}$; $6 \mathrm{~h}$.

Ref. Cohen, 761; BMCRE, 211; RIC, 171 a.

Mint of Rome (A.D. 201)

126. Obv. SEVERVS PIVS AVG.

Head of Septimus Severus, laureate, $r$.

Rev. PART. MAX. P. M. TR. P. VIIII.

Trophy and two captives.

Inv. No. 215

$S / W / D \quad 19$ mm.; 3.39 gr.; $6 \mathrm{~h}$.

Ref. Cohen, 370; BMCRE, 256; RIC, 176.

Mint of Rome (A.D. 202)

127. Obv. SEVERVS PIVS AVG.

Head of Septimus Severus, laureate, $r$.

Rev. $\quad$ PART. MAX. P. M. TR. P. X.

Trophy and two captives.

Inv. No. 223

$S / W / D \quad 19$ mm.; 3.52 gr.; lh.

Ref. Cohen, 372; BMCRE, 383; RIC, 184.

Mint of Rome (A.D. 200-202 ?)

128. Obv. SEVERVS AVG. PART. MAX.

Head of Septimus Severus, laureate, $r$.

Rev. IVNO REGINA

Juno, veiled standing l., holding patera and sceptre: at feet l., peacock.

Inv. No. 238

$S / W / D \quad 19$ mm.; 2.82 gr.; $12 \mathrm{~h}$.

Ref. ?.

Mint of Rome (A.D. 205)

129. $O b v$. SEVERVS PIVS AVG.

Head of Septimus Severus, laureate, $r$.

Rev. $\quad$ P. M. TR. P. XIII. COS. III. P. P.

Jupiter, naked, standing 1., holding thunderbolt and sceptre:at feet, 1., eagle. 
Inv. No. 213

$S / W / D \quad 19$ mm.; 2.78 gr.; $1 \mathrm{~h}$.

Ref. Cohen, 469; BMCRE, 471; RIC, 196.

\section{Mint of Rome (A.D. 206)}

130. $O b v$. SEVERVS PIVS AVG.

Head of Septimus Severus, laureate, $r$.

Rev. P. M. TR. P. XIIII. COS. III. P. P.

Annona standing l., holding corn-ears and cornucopiae: at feet, 1., modius.

Inv. No. 217

$S / W / D \quad 19 \mathrm{~mm}$.; $3.17 \mathrm{gr}$.; $7 \mathrm{~h}$.

Ref. Cohen, 476; BMCRE, 489; RIC, 200.

131.

$\begin{array}{ll} & \text { Similar } \\ \text { Inv. No. } & 234 \\ \text { S/W/D } & 19 \mathrm{~mm} . ; 3.51 \mathrm{gr} . ; 12 \mathrm{~h} . \\ \text { Ref. } & \text { Cohen, 476; BMCRE, 489; RIC, } 200 .\end{array}$

132.

Similar

Inv. No. 227

$S / W / D \quad 18 \mathrm{~mm}$; 3.36 gr.; $6 \mathrm{~h}$.

Ref. Cohen, 476; BMCRE, 489; RIC, 200.

133. Obv. SEVERVS PIVS AVG.

Head of Septimus Severus, laureate, $r$.

Rev. $\quad$ P. M. TR. P. XIIII. COS. III. P. P.

Genius standing 1., sacrificing out of patera over altar and holding cornears.

Inv. No. 208

$S / W / D \quad 20 \mathrm{~mm}$.; $3.82 \mathrm{gr}$.; $6 \mathrm{~h}$.

Ref. Cohen, 475; BMCRE, 493; RIC, 201.

Mint of Rome (A.D. 207)

134. Obv. SEVERVS PIVS AVG.

Head of Septimus Severus, laureate, $r$.

Rev. $\quad$ P. M. TR. P. XV. COS. III. P. P.

Inv. No. 226

$S / W / D \quad 19 \mathrm{~mm}$; $3.71 \mathrm{gr}$; $6 \mathrm{~h}$.

Ref. Cohen, 489; BMCRE, 534; RIC, 211. 


\section{Mint of Rome (A.D. 210)}

135. Obv. SEVERVS PIVS AVG.

Head of Septimus Severus, laureate, r.

Rev. P. M. TR. P. XVIII. COS. III. P. P.

Jupiter standing 1., holding thunderbolt and sceptre; by him, two children standing, one r., one 1 .

Inv. No. 201

$S / W / D \quad 19 \mathrm{~mm}$.; 3.45 gr.; $6 \mathrm{~h}$.

Ref. $\quad$ Cohen, 539; BMCRE, 18; RIC, 233.

136. Obv. SEVERVS PIVS AVG.

Head of Septimus Severus, laureate, $r$.

Rev. $\quad$ P. M. TR. P. XVIII. COS. III. P. P.

Neptün standing l., r. foot on globe, holding trident in l.hand.

Inv. No. 210

$S / W / D \quad 20 \mathrm{~mm}$; $3.61 \mathrm{gr}$; $6 \mathrm{~h}$.

Ref. Cohen, 543; BMCRE, 19; RIC, 234.

$137 . \quad$ Similar

Inv. No. 225

$S / W / D \quad 19 \mathrm{~mm}$.; 3.13 gr.; $12 \mathrm{~h}$.

Ref. Cohen, 543; BMCRE, 19; RIC, 234.

138. Obv. SEVERVS PIVS AVG.

Head of Septimus Severus, laureate, $r$.

Rev. P. M. TR. P. XVIII. COS. III. P. P.

Salus seated l., feeding snake held in her arms.

Inv. No. 230

$S / W / D \quad 19 \mathrm{~mm}$.; 3.26 gr.; $12 \mathrm{~h}$.

Ref. Cohen, 548; BMCRE, 20; RIC, 236.

Mint of Rome (A.D. 202-210)

139. Obv. SEVERVS PIVS AVG.

Head of Septimus Severus, laureate, $r$.

Rev. FORTUNA REDVX

Fortuna seated l., holding rudder and cornucopiae.

Inv. No. $\quad 29$

$S / W / D \quad 18 \mathrm{~mm}$; 3.27 gr.; lh.

Ref. Cohen, 181; BMCRE, 327; RIC, 264a.

140. $O b v$.

SEVERVS PIVS AVG.

Head of Septimus Severus, laureate, $r$. 
Rev. $\quad$ FVNDATOR PACIS

Septimus Severus veiled, standing l., holding branch and roll.

Inv. No. 218

$S / W / D \quad 18 \mathrm{~mm}$.; 3.45 gr.; $6 \mathrm{~h}$.

Ref. Cohen, 205; BMCRE, 330; RIC, 265.

141. Similar

Inv. No. 220

$S / W / D \quad 18 \mathrm{~mm}$.; 3.35 gr.; $6 \mathrm{~h}$.

Ref. Cohen, 205; BMCRE, 330; RIC, 265.

142. Obv. SEVERVS PIVS AVG.

Head of Septimus Severus, laureate, $r$.

Rev. INDVLGENTIA AVGG. IN CARTH.

The Dea Caelestis riding r., on a lion, holding thunderbolt and sceptre: below, waters gushing from rock.

Inv. No. 233

$S / W / D \quad 20 \mathrm{~mm}$.; 2.75 gr.; 4 h.

Ref. $\quad$ Cohen, 222; BMCRE, 335; RIC, 266.

143. Obv. SEVERVS PIVS AVG.

Head of Septimus Severus, laureate, $r$.

Rev. LIBERALITAS AVG. VI.

Liberalitas standing 1., holding account-board and cornucopiae.

Inv. No. 205

$S / W / D \quad 19$ mm.; 3.75 gr.; $7 \mathrm{~h}$.

Ref. Cohen, 298; BMCRE, 349; RIC, 278a.

144. $O b v$.

Head of Septimus Severus, laureate, $r$.

Rev. VICT. PART. MAX.

Victory advancing l., holding wreath and palm.

Inv. No. 235

$S / W / D \quad 19 \mathrm{~mm}$; $3.12 \mathrm{gr}$; $1 \mathrm{~h}$.

Ref. $\quad$ Cohen, 744; BMCRE, 367; RIC, 295.

145. $O b v$.

SEVERVS PIVS AVG.

Head of Septimus Severus, laureate, r.

Rev. $\quad$ VIRTVS AVGVSTOR

Roma seated 1., resting l., elbow on shield, holding Victory and parazonium.

Inv. No. 212

$S / W / D \quad 20 \mathrm{~mm}$.; 3.45 gr.; $1 \mathrm{~h}$. 
Ref. $\quad$ Cohen, 766; BMCRE, 373; RIC, 304.

\section{Mint of Emesa (A.D. 194-195)}

146. $O b v$.

IMP. CAE. L. SEP. SEV. PERT. AVG. COS. II.

Head of Septimus Severus, laureate, $r$.

Rev. MONET AVG.

Moneta standing 1. , holding scales and cornucopiae.

Inv. No. 241

$S / W / D \quad 17 \mathrm{~mm}$; 3.22 gr.; $12 \mathrm{~h}$.

Ref. Cohen, 330; BMCRE, 380; RIC, 411.

\section{Mint of Laodiceia (A.D. 196-197)}

147. Obv. L. SEPT. SEVER. PERT. AVG. IMP. VIII.

Head of Septimus Severus, laureate, $r$.

Rev. MONET AVG.

Moneta standing 1., holding scales and cornucopiae.

Inv. No. 202

$S / W / D \quad 18 \mathrm{~mm}$; 3.27 gr.; $6 \mathrm{~h}$.

Ref. $\quad$ Cohen, 332; BMCRE, 448; RIC, 484a.

148. Obv. L. SEPT. SEV. PERT. AVG. IMP. VIII.

Head of Septimus Severus, laureate, $r$.

Rev. $\quad$ P. M. TR. P. V. COS. II. P. P.

Pax seated 1., holding branch and sceptre.

Inv. No. 214

$S / W / D \quad 20 \mathrm{~mm}$.; 3.76 gr.; $12 \mathrm{~h}$.

Ref. Cohen, 444; BMCRE, 452; RIC, 490.

149. Obv. L. SEPT. SEV. PERT. AVG. IMP. VIIII.

Head of Septimus Severus, laureate, $r$.

Rev. PROFECTIO AVG.

Septimus Severus on horse pacing r., holding spear.

Inv. No. 246

$S / W / D \quad 15 \mathrm{~mm}$.; $3.42 \mathrm{gr}$; $12 \mathrm{~h}$.

Ref. Cohen, 580; BMCRE, 466; RIC, 494.

$150 . \quad$ Similar

Inv. No. 240

$S / W / D \quad 17$ mm.; 2.90 gr.; $6 \mathrm{~h}$.

Ref. Cohen, 580; BMCRE, 466; RIC, 494. 


\section{Julia Domna (Wife of Septimus Severus)} Mint of Rome (A.D. 193-196)

151. Obv. IVLIA DOMNA AVG.

Bust of Julia Domna, draped, r., hair waved and coiled at back.

Rev. VENERI VICTR.

Venus naked to waist, standing r., holding apple and palm, resting l. elbow on column.

Inv. No. 280

$S / W / D \quad 19 \mathrm{~mm}$.; $3.65 \mathrm{gr}$; $6 \mathrm{~h}$.

Ref. Cohen, 194; BMCRE, 49; RIC, 536.

152.

$\begin{array}{ll} & \text { Similar } \\ \text { Inv. No. } & 264 \\ \text { S/W/D } & 20 \mathrm{~mm} . ; \\ \text { Ref. } & \text { Cohen, 194; BMCRE, 12h. } \\ \end{array}$

Mint of Rome (A.D. 196-211)

153. Obv. IVLIA AVGVSTA

Bust of Julia Domna, draped, r., hair waved and coiled at back.

Rev. CERERI FRVGIF.

Ceres seated 1., holding corn-ears and torch.

Inv. No. 255

$S / W / D \quad 21 \mathrm{~mm} . ; 3.51 \mathrm{gr}$; $1 \mathrm{~h}$.

Ref. Cohen, 14; BMCRE, 10; RIC, 546.

154. Obv. IVLIA AVGVSTA

Bust of Julia Domna, draped, r., hair waved and coiled at back.

Rev. HILARITAS

Inv. No. 267

Hilaritas standing l., holding long palm and cornucopiae.

$S / W / D \quad 19 \mathrm{~mm}$.; $3.35 \mathrm{gr}$; $6 \mathrm{~h}$.

Ref. $\quad$ Cohen, 72; BMCRE, 31; RIC, 556.

155.

$\begin{array}{ll} & \text { Similar } \\ \text { Inv. No. } & 281 \\ \text { S/W/D } & 17 \mathrm{~mm} . ; \\ \text { Ref. } & \text { Cohen, 72; BMCRE, 31; RIC, } 556 .\end{array}$


156.

$\begin{array}{ll} & \text { Similar } \\ \text { Inv. No. } & 275 \\ \text { S/W/D } & 18 \mathrm{~mm} . ; 3.42 \text { gr.; } 6 \mathrm{~h} . \\ \text { Ref. } & \text { Cohen, 72; BMCRE, 31; RIC, } 556 .\end{array}$

157.

$\begin{array}{ll} & \text { Similar } \\ \text { Inv. No. } & 274 \\ \text { S/W/D } & 17 \mathrm{~mm} . ; 3.31 \mathrm{gr} . ; \text { 7h. } \\ \text { Ref. } & \text { Cohen, 72; BMCRE, 31; RIC, } 556 .\end{array}$

158. Obv. IVLIA AVGVSTA

Bust of Julia Domna, draped, r., hair waved and coiled at back.

Rev. IVNO

Juno, veiled standing l., holding patera and sceptre: at feet l., peacock.

Inv. No. 266

$S / W / D \quad 19$ mm.; 3.50 gr.; $12 \mathrm{~h}$.

Ref. $\quad$ Cohen, 82; BMCRE, 38; RIC, 559.

159. Obv. IVLIA AVGVSTA

Bust of Julia Domna, draped, r., hair waved and coiled at back.

Rev. IVNO REGINA

Juno, veiled standing l., holding patera and sceptre: at feet l., peacock.

Inv. No. 262

$S / W / D \quad 19$ mm.; 3.50 gr.; $12 \mathrm{~h}$.

Ref. $\quad$ Cohen, 97; BMCRE, 42; RIC, 560.

160. $O b v$ IVLIA AVGVSTA

Bust of Julia Domna, draped, r., hair waved and coiled at back.

Rev. PIETAS AVGG.

Pietas, veiled, standing l., dropping incense on altar 1. and holding box.

Inv. No. 259

$S / W / D \quad 18 \mathrm{~mm} . ; 3.25$ gr.; $12 \mathrm{~h}$.

Ref. $\quad$ Cohen, 150; BMCRE, 62; RIC, 572.

$161 . \quad$ Similar

Inv. No. 257

$S / W / D \quad 20 \mathrm{~mm}$.; 3.28 gr.; $12 \mathrm{~h}$.

Ref. $\quad$ Cohen, 150; BMCRE, 62; RIC, 572.

162.

$\begin{array}{ll} & \text { Similar } \\ \text { Inv. No. } & 254 \\ \text { S/W/D } & 21 \mathrm{~mm} . ; 3.63 \text { gr.; 12h. }\end{array}$


Ref. Cohen, 150; BMCRE, 62; RIC, 572.

163. Obv. IVLIA AVGVSTA

Bust of Julia Domna, draped, r., hair waved and coiled at back.

Rev. PIETAS PVBLICA.

Inv. No. 258

$S / W / D \quad 20 \mathrm{~mm}$; $3.34 \mathrm{gr}$; $12 \mathrm{~h}$.

Ref. Cohen, 156; BMCRE, 69; RIC, 574.

164.

$\begin{array}{ll} & \text { Similar } \\ \text { Inv. No. } & 269 \\ \text { S/W/D } & 20 \mathrm{~mm} . ; 3.25 \text { gr.; 12h. } \\ \text { Ref. } & \text { Cohen, 156; BMCRE, 69; RIC, } 574 .\end{array}$

165.

Inv. No. 279

$S / W / D \quad 20 \mathrm{~mm}$.; 3.33 gr.; $6 \mathrm{~h}$.

Ref. Cohen, 156; BMCRE, 69; RIC, 574.

166. Obv. IVLIA AVGVSTA

Bust of Julia Domna, draped, r., hair waved and coiled at back.

Rev. PVDICITIA.

Inv. No 273

$S / W / D \quad 18 \mathrm{~mm} . ; 3.36$ gr.; $12 \mathrm{~h}$.

Ref. $\quad$ Cohen, 168; BMCRE, 72; RIC, 576.

167. Obv. IVLIA AVGVSTA

Rev. SAECVLI FELICITAS

Isis, wearing peaked head-dress, standing r., l. foot on prow, with the infant Horus at her breast: to l., altar, against which rests a rudder.

Inv. No. 260

$S / W / D \quad 19$ mm.; 3.35 gr.; $7 \mathrm{~h}$.

Ref. Cohen, 174; BMCRE, 75; RIC, 577.

$168 . \quad$ Similar

Inv. No. 265

$S / W / D \quad 19 \mathrm{~mm} . ; 3.33$ gr.; $12 \mathrm{~h}$.

Ref. Cohen, 174; BMCRE, 75; RIC, 577. 
169. Obv. IVLIA AVGVSTA

Bust of Julia Domna, draped, r., hair waved and coiled at back.

Rev. $\quad$ VENVS FELIX

Venus standing front, head 1., holding apple and drawing drapery from shoulder.

Inv. No. 277

$S / W / D \quad 19 \mathrm{~mm}$.; $2.71 \mathrm{gr}$; $6 \mathrm{~h}$.

Ref. $\quad$ Cohen, 198; BMCRE, 85; RIC, 580.

170.

\begin{tabular}{ll} 
& Similar \\
Inv. No. & 271 \\
S/W/D & $18 \mathrm{~mm} . ;$ \\
Ref. & Cohen, 198; BMC.; 12h. \\
\hline
\end{tabular}

\section{Mint of Rome (A.D. 211-217)}

171. $O b v$.

\section{IVLIA PIA FELIX AVG.}

Bust of Julia Domna, draped, r., hair waved and coiled at back.

Rev. DIANA LVCIFERA

Diana standing l., holding torch in both hands.

Inv. No. 253

$S / W / D \quad 20 \mathrm{~mm}$.; 3.53 gr.; $1 \mathrm{~h}$.

Ref. Cohen, 32; BMCRE, 1; RIC, 373a.

172.

$\begin{array}{ll} & \text { Similar } \\ \text { Inv. No. } & 268 \\ \text { S/W/D } & 18 \mathrm{~mm} . ; \text { 3.21 gr.; lh. } \\ \text { Ref. } & \text { Cohen, 32; BMCRE, 1; RIC, 373a. }\end{array}$

173.

$\begin{array}{ll} & \text { Similar } \\ \text { Inv. No. } & 272 \\ \text { S/W/D } & 18 \mathrm{~mm} . ; \text { 2.76 gr.; 7h. } \\ \text { Ref. } & \text { Cohen, 32; BMCRE, 1; RIC, 373a. }\end{array}$

174.

Similar

Inv. No. 276

$S / W / D \quad 19 \mathrm{~mm}$; $3.19 \mathrm{gr}$; $12 \mathrm{~h}$.

Ref. Cohen, 32; BMCRE, 1; RIC, 373a.

175.

$\begin{array}{ll} & \text { Similar } \\ \text { Inv. No. } & 278 \\ \text { S/W/D } & 19 \mathrm{~mm} . ; 3.15 \text { gr.; 6h. } \\ \text { Ref. } & \text { Cohen, 32; BMCRE, 1; RIC, 373a. }\end{array}$


176. $O b v$. IVLIA PIA FELIX AVG.

Bust of Julia Domna, draped, r., hair waved and coiled at back.

Rev. MAT. AVGG. MAT. SEN. M. PATR.

Julia Domna seated 1., holding branch and sceptre.

Inv. No. 256

$S / W / D \quad 19$ mm.; 3.48 gr.; lh.

Ref. Cohen, 111; BMCRE, 11A; RIC, 381.

177. Obv. IVLIA PIA FELIX AVG.

Bust of Julia Domna, draped, r., hair waved and coiled at back.

Rev. VESTA

Vesta standing 1., holding palladium and sceptre..

Inv. No. 263

$S / W / D \quad 19$ mm.; 3.20 gr.; $6 \mathrm{~h}$.

Ref. Cohen, 230; BMCRE, 11A; RIC, 390.

Mint of Laodiceia (A.D. 196-202)

178. Obv. IVLIA AVGVSTA

Bust of Julia Domna, draped, r., hair waved and coiled at back.

Rev. CERERI FRVGIF.

Ceres seated 1., holding corn-ears and torch.

Inv. No. 261

$S / W / D \quad 20 \mathrm{~mm}$; 3.43 gr.; $12 \mathrm{~h}$.

Ref. Cohen, 14; BMCRE, 592; RIC, 636.

Caracalla, As Caesar (A.D. 196-198)

Mint of Rome (A.D. 196)

179. Obv. M. AVR. ANTONINVS CAES.

Bust of Caracalla, draped, cuirassed, head, bare, r.

Rev. SPEI PERPETVAE

Inv. No. 302

$S / W / D \quad 19 \mathrm{~mm}$; 3.22 gr.; $7 \mathrm{~h}$.

Ref. Cohen, 594 ; BMCRE, 190; RIC, 5.

Mint of Rome (A.D. 196-198)

180. Obv. M. AVR. ANTON. CAES. PONTIF.

Bust of Caracalla, draped, cuirassed, head, bare, $r$.

Rev. DESTINATO IMPERAT.

Lituus, apex, bucranium, simpulum. 
Inv. No. $\quad 317$

$S / W / D \quad 17 \mathrm{~mm}$.; 3.46 gr.; $7 \mathrm{~h}$.

Ref. Cohen, 53; BMCRE, 193; RIC, 6.

181.

$\begin{array}{ll}\text { Inv. No. } & 318 \\ \text { S/W/D } & 16 \mathrm{~mm} . ; 3.27 \text { gr.; 7h. } \\ \text { Ref. } & \text { Cohen, 53; BMCRE, 193; RIC, } 6 .\end{array}$

182. Obv. M. AVR. ANTON. CAES. PONTIF.

Bust of Caracalla, draped, cuirassed, head, bare, r.

Rev. MARTI VLTORI

Mars advancing r.,holding spear and trophy..

Inv. No. 312

$S / W / D \quad 17 \mathrm{~mm}$.; 3.09 gr.; $12 \mathrm{~h}$.

Ref. Cohen, 154; BMCRE, 202; RIC, 11.

Caracalla, As Augustus (A.D. 198-217)

Mint of Rome (A.D. 199-200)

183. Obv. ANTONINVS AVGVSTVS.

Bust of Caracalla, laureate, draped, cuirassed, r.

Rev. $\quad$ SEVERI P. II. AVG. FIL.

Caracalla in military dress, standing 1., holding Victory on globe and spear: to l., captive seated.

Inv. No. $\quad 311$

$S / W / D \quad 18 \mathrm{~mm} . ; 3.58 \mathrm{gr}$; $6 \mathrm{~h}$.

Ref. $\quad$ Cohen, 590; BMCRE, 172; RIC, 45.

Mint of Rome (A.D. 202)

184. Obv. ANTONINVS PIVS AVG.

Bust of Caracalla, laureate draped, $r$.

Rev. VOT. SVSC. DEC. PON. TR. P. V. COS.

Caracalla standing l., sacrificing out of patera over altar and holding book.

Inv. No. 286

$S / W / D \quad 19$ mm.; 3.67 gr.; $6 \mathrm{~h}$.

Ref. $\quad$ Cohen, 686; BMCRE, 396; RIC, 68.

Mint of Rome (A.D. 205)

185. $O b v$.

ANTONINVS PIVS AVG.

Bust of Caracalla, laureate draped, $r$.

Rev. PONTIF TR. P. VIII. COS. II. 
Salus seated 1., feeding snake coiled round altar, l. arm on side of chair.

Inv. No. 297

$S / W / D \quad 19$ mm.; 3.58 gr.; $6 \mathrm{~h}$.

Ref. $\quad$ Cohen, 422; BMCRE, 484; RIC, 82.

Mint of Rome (A.D. 206)

186. Obv. ANTONINVS PIVS AVG.

Bust of Caracalla, laureate draped, $r$.

Rev. $\quad$ PONTIF TR. P. VIIII. COS. II.

Mars in military dress, standing l., resting $\mathrm{r}$. hand on shield and holding spear.

Inv. No. 296

$S / W / D \quad 20 \mathrm{~mm}$.; 3.49 gr.; $6 \mathrm{~h}$.

Ref. $\quad$ Cohen, 424; BMCRE, 497; RIC, 83.

187.

$\begin{array}{ll} & \text { Similar } \\ \text { Inv. No. } & 289 \\ \text { S/W/D } & 19 \mathrm{~mm} . ; 3.27 \text { gr.; 12h. } \\ \text { Ref. } & \text { Cohen, 424; BMCRE, 497; RIC, } 83 .\end{array}$

Mint of Rome (A.D. 207)

188. $O b v$.

ANTONINVS PIVS AVG.

Head of Caracalla, laureate, $r$.

Rev. PONTIF TR. P. X. COS. II.

Mars,with cloak flying, advancing r., holding spear and trophy.

Inv. No. 299

$S / W / D \quad 20$ mm.; 3.13 gr.; $6 \mathrm{~h}$.

Ref. $\quad$ Cohen, 431; BMCRE, 542; RIC, 88.

189. Obv. ANTONINVS PIVS AVG.

Head of Caracalla, laureate, $r$.

Rev. $\quad$ PONTIF TR. P. X. COS. II.

Securitas seated r., propping head on $\mathrm{r}$. hand holding sceptre: in front, altar.

Inv. No. 309

$S / W / D \quad 18 \mathrm{~mm}$; 3.32 gr.; $1 \mathrm{~h}$.

Ref. $\quad$ Cohen, 434; BMCRE, 549; RIC, 92.

Mint of Rome (A.D. 209)

190. $O b v$.

ANTONINVS PIVS AVG.

Head of Caracalla, laureate, $r$.

Rev. $\quad$ PONTIF TR. P. XII. COS. III. 
Virtus helmeted, standing r., l.foot on helmet, holding spear and parazonium.

Inv. No. 288

$S / W / D \quad 19$ mm.; 3.57 gr.; $6 \mathrm{~h}$.

Ref. $\quad$ Cohen, 464; BMCRE, 13; RIC, 112.

Mint of Rome (A.D. 210)

191. $O b v$. ANTONINVS PIVS AVG. BRIT.

Head of Caracalla, laureate, $r$.

Rev. PONTIF TR. P. XIII. COS. III.

Virtus helmeted, standing r., l.foot on helmet, holding spear and parazonium.

Inv. No. 290

$S / W / D \quad 19$ mm.; 3.25 gr.; $6 \mathrm{~h}$.

Ref. $\quad$ Cohen, 478; BMCRE, 37; RIC, 117.

192.

$\begin{array}{ll} & \text { Similar } \\ \text { Inv. No. } & 305 \\ \text { S/W/D } & 18 \mathrm{~mm} . ; 3.82 \text { gr.; 12h. } \\ \text { Ref. } & \text { Cohen, 478; BMCRE, 37; RIC, } 117 .\end{array}$

193. Similar

Inv. No. 282

$S / W / D \quad 19 \mathrm{~mm}$.; 3.50 gr.; $12 \mathrm{~h}$.

Ref. Cohen, 478; BMCRE, 37; RIC, 117.

194. Obv. ANTONINVS PIVS AVG.

Head of Caracalla, laureate, $r$.

Rev. VIRTVS AVGG.

Caracalla, in military dress, standing front, head r., holding spear and parazonium: to l., rivergood reclining, to $\mathrm{r}$., two captives.

Inv. No. 283

$S / W / D \quad 19$ mm.; 2.97 gr.; $12 \mathrm{~h}$.

Ref. Cohen, 670; BMCRE, 520; RIC, 175.

195. Obv. ANTONINVS PIVS AVG.

Head of Caracalla, laureate, $r$.

Rev. VIRTVS AVGVSTOR

Virtus, helmeted, seated 1., holding Victory and parazonium: behind, shield.

Inv. No. 285

$S / W / D \quad 19 \mathrm{~mm}$; 3.19 gr.; $7 \mathrm{~h}$. 
Ref. $\quad$ Cohen, 672; BMCRE, 522; RIC, 176.

196. Obv. ANTONINVS PIVS AVG.

Head of Caracalla, laureate, $r$.

Rev. VOTA SVS. CEPTA X.

Caracalla, veiled, standing l., sacrificing out of patera over tripod and holding roll.

Inv. No. 294

$S / W / D \quad 21 \mathrm{~mm}$; 3.57 gr.; $12 \mathrm{~h}$.

Ref. Cohen, 689; BMCRE, 524; RIC, 179.

197.

$\begin{array}{ll} & \text { Similar } \\ \text { Inv. No. } & 304 \\ \text { S/W/D } & 19 \mathrm{~mm} . ; 3.20 \mathrm{gr} . ; 12 \mathrm{~h} . \\ \text { Ref. } & \text { Cohen, 689; BMCRE, 524; RIC, } 179 .\end{array}$

\section{Mint of Rome (A.D. 210-213)}

198. $O b v$.

ANTONINVS PIVS AVG. BRIT.

Head of Caracalla, laureate, $r$.

Rev. MARTI PROPVGNATORI

Mars hurrying l., holding spear and trophy.

Inv. No. 300

$S / W / D \quad 20 \mathrm{~mm}$.; 3.17 gr.; $12 \mathrm{~h}$.

Ref. Cohen, 150; BMCRE, 87; RIC, 223.

199. Obv. ANTONINVS PIVS AVG. BRIT.

Head of Caracalla, laureate, $r$.

Rev. MONETA AVG.

Moneta standing l., holding scales and cornucopiae.

Inv. No. 293

$S / W / D \quad 19$ mm.; 2.93 gr.; $7 \mathrm{~h}$.

Ref. Cohen, 165; BMCRE, 90; RIC, 224.

200.

$\begin{array}{ll} & \text { Similar } \\ \text { Inv. No. } & 298 \\ \text { S/W/D } & 19 \mathrm{~mm} . ; 3.49 \mathrm{gr} . ; 6 \mathrm{~h} . \\ \text { Ref. } & \text { Cohen, 165; BMCRE, 90; RIC, 224. }\end{array}$

201.

$\begin{array}{ll} & \text { Similar } \\ \text { Inv. No. } & 291 \\ \text { S/W/D } & 18 \mathrm{~mm} . ; 3.16 \mathrm{gr} . ; 12 \mathrm{~h} . \\ \text { Ref. } & \text { Cohen, 165; BMCRE, 90; RIC, } 224 .\end{array}$


202.

$\begin{array}{ll} & \text { Similar } \\ \text { Inv. No. } & 287 \\ \text { S/W/D } & 19 \mathrm{~mm} . ; 3.33 \text { gr.; 12h. } \\ \text { Ref. } & \text { Cohen, 165; BMCRE, 90; RIC, } 224 .\end{array}$

203.

Inv. No. 284

$S / W / D \quad 19 \mathrm{~mm}$.; $3.28 \mathrm{gr}$; $6 \mathrm{~h}$.

Ref. Cohen, 165; BMCRE, 90; RIC, 224.

204. Obv. ANTONINVS PIVS AVG. BRIT.

Head of Caracalla, laureate, $r$.

Rev. PROFECTIO AVG.

Caracalla in military dress standing r., holding spear: behind, two standarts.

Inv. No. 308

$S / W / D \quad 19 \mathrm{~mm}$.; 3.08 gr.; $6 \mathrm{~h}$.

Ref. Cohen, 508; BMCRE, 97; RIC, 225.

205. Obv. ANTONINVS PIVS AVG. BRIT.

Head of Caracalla, laureate, $r$.

Rev. VICTORIAE BRIT.

Victory hurrying r., carrying trophy in both hands.

Inv. No. 303

$S / W / D \quad 20 \mathrm{~mm}$.; $3.28 \mathrm{gr}$; $6 \mathrm{~h}$.

Ref. Cohen, 629; BMCRE, 102; RIC, $231 \mathrm{~A}$.

\section{Mint of Rome (A.D. 214)}

206. Obv. ANTONINVS PIVS AVG. GERM.

Head of Caracalla, laureate, $r$.

Rev. $\quad$ P. M. TR. P. XVII. COS. IIII. P. P.

Jupiter, bare to waist, standing l., holding thunderbolt and sceptre:

at feet, eagle.

Inv. No. 307

$S / W / D \quad 19$ mm.; 3.18 gr.; $6 \mathrm{~h}$.

Ref. Cohen, 239; BMCRE, 94; RIC, 240.

207. Obv. ANTONINVS PIVS AVG. GERM.

Head of Caracalla, laureate, $r$.

Rev. $\quad$ P. M. TR. P. XVII. COS. IIII. P. P.

Caracalla standing 1., holding branch and baton.

Inv. No. 306

$S / W / D \quad 18 \mathrm{~mm}$; 3.10 gr.; $12 \mathrm{~h}$. 
Ref. $\quad$ Cohen, 247; BMCRE, ?; RIC, 246.

Mint of Rome (A.D. 215)

208. $O b v$. ANTONINVS PIVS AVG. GERM.

Head of Caracalla, laureate, $r$.

Rev. P. M. TR. P. XVIII. COS. IIII. P. P.

Apollon laureate in long dress, standing 1., holding branch and leaning on spear.

Inv. No. 292

$S / W / D \quad 20$ mm.; 2.95 gr.; $12 \mathrm{~h}$.

Ref. Cohen, 284; BMCRE,? RIC, 255.

209. $O b v$. ANTONINVS PIVS AVG. GERM.

Mint of Rome (A.D. 217)

Head of Caracalla, laureate, $r$.

Rev. $\quad$ P. M. TR. P. XX. COS. IIII. P. P.

Jupiter standing front, head l., holding thunderbolt and sceptre.

Inv. No. 301

$S / W / D \quad 18 \mathrm{~mm}$; 3.62 gr.; $1 \mathrm{~h}$.

Ref. $\quad$ Cohen, 373 (similar); BMCRE, 179; RIC, 286.

210. Obv. ANTONINVS PIVS AVG. GERM.

Head of Caracalla, laureate, $r$.

Rev. INDVLGENTIAE AVG.

Indulgentia seated l., holding patera and sceptre.

Inv. No. $\quad 310$

$S / W / D \quad 18 \mathrm{~mm}$.; $3.23 \mathrm{gr}$.; $7 \mathrm{~h}$.

Ref. Cohen, 103; BMCRE, 68; RIC, 300.

211. Obv. ANTONINVS PIVS AVG. GERM.

Head of Caracalla, laureate, $r$.

Rev. VENVS VICTRIX

Venüs standing l., holding Victory and spear and leaning on shield set on helmet.

Inv. No. 295

$S / W / D \quad 20 \mathrm{~mm}$.; 2.85 gr.; $12 \mathrm{~h}$.

Ref. $\quad$ Cohen, 606; BMCRE, 82; RIC, $311 \mathrm{lb}$.

Mint of Laodiceia (A.D. 196-197)

212. Obv. M. AVR. ANTON. CAES. PONTIF

Bust of Caracalla, draped, cuirassed, head, bare, r.. 
Rev. SECVRITAS PERPETVA

Minerva standing l, with aegis on breast, resting $r$. hand on shield and holding spear.

Inv. No. $\quad 315$

$S / W / D \quad 18$ mm.; 2.99 gr.; $6 \mathrm{~h}$.

Ref. Cohen, 566 (similar); BMCRE, 459; RIC, 330.

\section{Mint of Rome (A.D. 198)}

213. Obv. IMP. CAE. M. AVR. ANT. AVG. P. TR. P.

Bust of Caracalla, laureate,draped, $r$.

Rev. $\quad$ MINER VICTRIX

Minerva standing 1., holding Victory and spear: at feet, shield: behind trophy.

Inv. No. $\quad 314$

$S / W / D \quad 18 \mathrm{~mm}$.; 3.16 gr.; $6 \mathrm{~h}$.

Ref. Cohen, 159; BMCRE, ?; RIC, 336.

$214 . \quad$ Similar

Inv. No. 313

$S / W / D \quad 20$ mm.; 3.54 gr.; $12 \mathrm{~h}$.

Ref. $\quad$ Cohen, 159; BMCRE, ?; RIC, 336.

\section{Mint of Rome (A.D. 198)}

215. Obv. IMP. CAE. M. AVR. ANT. AVG. P.TR. P.

Bust of Caracalla, laureate,draped, $r$.

Rev. SPES PVBLICA

Inv. No. $\quad 316$

$S / W / D \quad 17 \mathrm{~mm} . ; 3.43$ gr.; $12 \mathrm{~h}$

Ref. $\quad$ Cohen, 599; BMCRE, ?; RIC, 338b.

\section{Plautilla (Wife of Garacalla)}

Mint of Rome (A.D. 202-205)

216. Obv. PLAVTILLAE AVGVSTAE

Bust of Plautilla, draped, $r$.

Rev. PROPAGO IMPERI

Inv. No. 321

$S / W / D \quad 19 \mathrm{~mm}$.; $3.51 \mathrm{gr}$.; $6 \mathrm{~h}$.

Ref. Cohen, 21; BMCRE, 406; RIC, 362. 
217. $O b v$ PLAVTILLAE AVGVSTA

Bust of Plautilla, draped, r.

Rev. PIETAS AVG.

Inv. No. 320

$S / W / D \quad 20 \mathrm{~mm}$.; 2.83 gr.; $6 \mathrm{~h}$.

Ref. Cohen, 16 (Rev. Plautilla ?); BMCRE, 422; RIC, 367.

218. Obv. PLAVTILLAE AVGVSTA

Bust of Plautilla, draped, r.

Rev. VENVS VICTRIX

Venus, bare to waist, standing l., holding apple and palm and resting left elbow on shield: at her feet, 1., Cupid.

Inv. No. $\quad 319$

$S / W / D \quad 21 \mathrm{~mm}$; 3.49; $6 \mathrm{~h}$.

Ref. $\quad$ Cohen, 25; BMCRE, 428; RIC, 369.

219.

Similar

Inv. No. 270

$S / W / D \quad 19 \mathrm{~mm}$.; $3.23 \mathrm{gr}$; $7 \mathrm{~h}$.

Ref. $\quad$ Cohen, 25; BMCRE, 428; RIC, 369.

Geta, As Caesar (A.D. 198-209)

Mint of Rome (A.D. 198-200)

220. Obv. L. SEPTIMVS GETA CAES.

Bust of Geta, draped, head bare, $r$.

Rev. FELICITAS TEMPOR

Felicitas standing r., holding long caduceus in 1 . hand, clasping r. hands with Geta, standing l., holding cornucopiae.

Inv. No. $\quad 337$

$S / W / D \quad 18 \mathrm{~mm}$; $3.19 \mathrm{gr}$; $12 \mathrm{~h}$.

Ref. Cohen, 49; BMCRE, 146; RIC, 1.

221. Obv. L. SEPTIMVS GETA CAES.

Bust of Geta, draped, head bare, r.

Rev. $\quad$ FELICITAS TEMPOR

Felicitas standing 1., holding caduceus and cornucopiae.

Inv. No. 338

$S / W / D \quad 18$ mm.; 3.22 gr.; $12 \mathrm{~h}$.

Ref. Cohen, 44; BMCRE, 144; RIC, 2.

222.

Similar

Inv. No. 333 
$S / W / D \quad 18 \mathrm{~mm}$.; $3.28 \mathrm{gr}$.; $5 \mathrm{~h}$.

Ref. Cohen, 44; BMCRE, 144; RIC, 2.

223. Obv. L. SEPTIMVS GETA CAES.

Bust of Geta, draped, head bare, $r$.

Rev. SEVERI. PII. AVG. FIL.

Lituus, knife, jug, simpulum,sprinkler.

Inv. No. $\quad 340$

$S / W / D \quad 18 \mathrm{~mm}$.; 3.16 gr.; $6 \mathrm{~h}$.

Ref. $\quad$ Cohen, 188; BMCRE, 147; RIC, 3.

224. Obv. L. SEPTIMVS GETA CAES.

Bust of Geta, draped, head bare, $r$.

Rev. SPES PVBLICA

Spes advancing 1 ., holding flower and raising skirt.

Inv. No. $\quad 331$

$S / W / D \quad 18 \mathrm{~mm}$.; 3.37 gr.; $12 \mathrm{~h}$.

Ref. Cohen, 193; BMCRE, 149; RIC, 4.

Mint of Rome (A.D. 200-202)

225. Obv. P. SEPT. GETA CAES. PONT.

Bust of Geta, draped, head bare, $r$.

Rev. FELICITAS AVGG.

Felicitas standing 1., holding caduceus and cornucopiae.

Inv. No. 334

$S / W / D \quad 19 \mathrm{~mm}$.; 3.59 gr.; $6 \mathrm{~h}$.

Ref. Cohen, 36; BMCRE, 218; RIC, 8.

226. Obv. P. SEPT. GETA CAES. PONT.

Bust of Geta, draped, head bare, $r$.

Rev. FELICITAS PVBLICA

Felicitas standing 1., holding caduceus and cornucopiae.

Inv. No. 336

$S / W / D \quad 19$ mm.; 3.55 gr.; $6 \mathrm{~h}$.

Ref. Cohen, 38; BMCRE, 220; RIC, 9a.

227. Obv. P. SEPT. GETA CAES. PONT.

Bust of Geta, draped, head bare, $r$.

Rev. NOBILITAS

Nobilitas standing r., holding sceptre and palladium.

Inv. No. 335

$S / W / D \quad 18 \mathrm{~mm}$; $3.57 \mathrm{gr}$; $6 \mathrm{~h}$.

Ref. Cohen, 90; BMCRE, 223; RIC, 13a. 
228. Obv. P. SEPT. GETA CAES. PONT.

Bust of Geta, draped, head bare, r.

Rev. PRINC. IVVENT.

Inv. No. $\quad 326$

$S / W / D \quad 20$ mm.; 3.34 gr.; $6 \mathrm{~h}$.

Ref. Cohen, 159; BMCRE, 229; RIC, 15a.

229. Obv. P. SEPT. GETA CAES. PONT.

Bust of Geta, draped, head bare, $r$.

Rev. PRINC. IVVENT.

Geta standing l., holding branch and spear: behind trophy.

Inv. No. 332

$S / W / D \quad 19$ mm.; 3.30 gr.; 12h.

Ref. Cohen, 156; BMCRE,?; RIC, 16a.

230. Obv. P. SEPT. GETA CAES. PONT.

Bust of Geta, draped, head bare, r.

Rev. PRINC. IVVENTVTIS.

Inv. No. 327

$S / W / D \quad 21 \mathrm{~mm}$; $3.32 \mathrm{gr}$; $6 \mathrm{~h}$.

Ref. $\quad$ Cohen, 157; BMCRE, 234; RIC, 18.

231.

Inv. No. 339

$S / W / D \quad 18 \mathrm{~mm}$.; 3.35 gr.; $6 \mathrm{~h}$.

Ref. $\quad$ Cohen, 157; BMCRE, 234; RIC, 18.

232. Obv. P. SEPT. GETA CAES. PONT.

Bust of Geta, draped,cuirassed, head bare, $r$.

Rev. SECVRIT IMPER II.

Securitas seated l., holding globe, l. arm on chair.

Inv. No. 329

$S / W / D \quad 19$ mm.; 3.52 gr.; lh.

Ref. Cohen, 183; BMCRE, 240; RIC, $20 \mathrm{~b}$.

233. Obv. P. SEPT. GETA CAES. PONT.

Bust of Geta, draped,cuirassed, head bare, $r$.

Rev. VICT. AETERN.

Victory flying l., holding in both hands wreath over shield on low base.

Inv. No. 328

$S / W / D \quad 19$ mm.; 3.35 gr.; $6 \mathrm{~h}$. 
Ref. $\quad$ Cohen, 206; BMCRE, 247; RIC, 23.

\section{Mint of Rome (A.D. 203-208)}

234. Obv. GETA CAES. PONT. COS.

Bust of Geta, draped, head bare, $r$.

Rev.. $\quad$ FELICITAS AVGG.

Felicitas standing 1., holding caduceus and cornucopiae.

Inv. No. $\quad 330$

$S / W / D \quad 19$ mm.; 3.58 gr.; $12 \mathrm{~h}$.

Ref. Cohen, 35; BMCRE, 438; RIC, 29.

235. Obv. P. SEPTIMVS GETA CAES.

Bust of Geta, draped, head bare, r.

Rev.. $\quad$ PROVID DEORVM

Providentia standing l., holding wand over globe and sceptre.

Inv. No. 322

$S / W / D \quad 21 \mathrm{~mm}$.; 3.42 gr.; $12 \mathrm{~h}$.

Ref. Cohen, 170; BMCRE, 458; RIC, 51.

Mint of Rome (A.D. 209)

236. Obv. P. SEPTIMVS GETA CAES.

Bust of Geta, draped, head bare, $r$.

Rev. $\quad$ PONTIF COS. II.

Genius, naked, standing l., sacrificing out of patera over lighted altar and holding corn-ears.

Inv. No. 325

$S / W / D \quad 20 \mathrm{~mm}$.; 3.27 gr.; $12 \mathrm{~h}$.

Ref. Cohen, 114; BMCRE, 579; RIC, 59a.

237. Obv. P. SEPTIMVS GETA CAES.

Bust of Geta, draped, head bare, $r$.

Rev. PONTIF COS. II.

Inv. No. 323

$S / W / D \quad 20$ mm.; 2.52 gr.; $1 \mathrm{~h}$.

Ref. Cohen, 117; BMCRE, ?; RIC, 6la

238. Obv. P. SEPTIMVS GETA CAES.

Bust of Geta, draped, head bare, $r$.

Rev. $\quad$ PONTIF COS. II.

Geta, veiled, standing 1., sacrificing out of patera over tripod and holding short sceptre. 


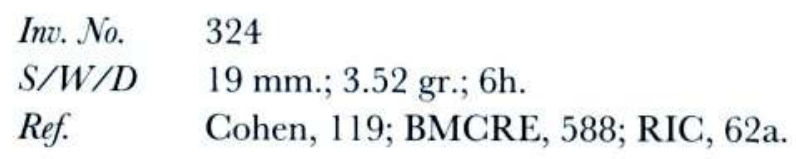

Elagabalus (A.D. 218-222)

Mint of Rome (A.D. 218)

239. Obv. IMP. CAES. M. AVR. ANTONINVS AVG.

Bust of Elagabalus, radiate, draped and cuirassed $r$.

Rev. $\quad$ P. M. TR. P. COS. P. P. .

Roma seated 1., holding Victory and sceptre; by her side, shield.

Inv. No. 341

$S / W / D \quad 25 \mathrm{~mm}$; 4.17 gr.; $12 \mathrm{~h}$.

Ref. Cohen, 126; BMCRE, 3; RIC, 1.

Mint of Rome (A.D. 220-221)

240. Obv. IMP. ANTONINVS PIVS. AVG.

Bust of Elagabalus, laureate, draped and cuirassed $r$.

Rev. P. M. TR. P. IIII COS. III. P. P. .

Elagabalus standing l., sacrificing over lighted altar, holding patera in r., branch in l., in field, star.

Inv. No. 345

$S / W / D \quad 20 \mathrm{~mm}$.; 3.23 gr.; $12 \mathrm{~h}$.

Ref. Cohen, 208; BMCRE, 256; RIC, 46.

Mint of Rome (A.D. 222)

241. Obv. IMP. CAES. ANTONINVS AVG.

Bust of Elagabalus, laureate, draped and cuirassed $r$.

Rev. MARS VICTOR.

Mars, nude, with flowing cloak, advancing r., holding spear and trophy. Inv. No. 344

$S / W / D \quad 20 \mathrm{~mm}$.; $2.94 \mathrm{gr}$; $12 \mathrm{~h}$.

Ref. Cohen, 110; BMCRE, 110; RIC, 120.

242. Obv. IMP. CAES. M. AVR. ANTONINVS AVG.

Bust of Elagabalus, radiate, draped and cuirassed $r$.

Rev. SALVS ANTONINI AVG.

Salus standing r., feeding a snake which she holds inher arms.

Inv. No. 342

$S / W / D \quad 23 \mathrm{~mm}$.; 5.56 gr.; $12 \mathrm{~h}$.

Ref. Cohen, 255; BMCRE, 23; RIC, 138. 
243. Obv. IMP. CAES. M. AVR. ANTONINVS AVG.

Bust of Elagabalus, laureate, draped and cuirassed $r$.

Rev. $\quad$ VICTOR. ANTONINI AVG.

Victory running r., holding wreath and palm.

Inv. No. 347

$S / W / D \quad 20 \mathrm{~mm} . ; 3.15$ gr.; $12 \mathrm{~h}$.

Ref. $\quad$ Cohen, 289; BMCRE, 36; RIC, 156.

244. Obv. IMP. CAES. M. AVR. ANTONINVS AVG.

Bust of Elagabalus, radiate, draped and cuirassed $r$.

Rev. VICTOR. ANTONINI AVG.

Victory running $\mathrm{r}$., holding wreath and palm.

Inv. No. 343

$S / W / D \quad 22$ mm.; 4.95 gr.; $12 \mathrm{~h}$.

Ref. $\quad$ Cohen, 291; BMCRE, 32; RIC, 155.

245. Obv. IMP. ANTONINVS PIVS. AVG.

Bust of Elagabalus, laureate, draped and cuirassed $r$.

Rev. VICTORIA AVG.

Victory flying l., holding diadem in both hands; no each side, a small shield; in field, star.

Inv. No. 346

$S / W / D \quad 20 \mathrm{~mm}$; 3.35 gr.; $12 \mathrm{~h}$.

Ref. Cohen, 300; BMCRE, 234; RIC, 161.

Mint of Antiocheia (A.D. 222)

246. $O b v$ IMP. ANTONINVS AVG.

Bust of Elagabalus, laureate, draped and cuirassed $r$.

Rev. SPEI PERPETVAE

Spes advancing l., holding flower and raising skirt.

Inv. No. 348

$S / W / D \quad 18 \mathrm{~mm}$.; $3.91 \mathrm{gr}$.; $7 \mathrm{~h}$.

Ref. Cohen, 273; BMCRE, 313; RIC, 199. 


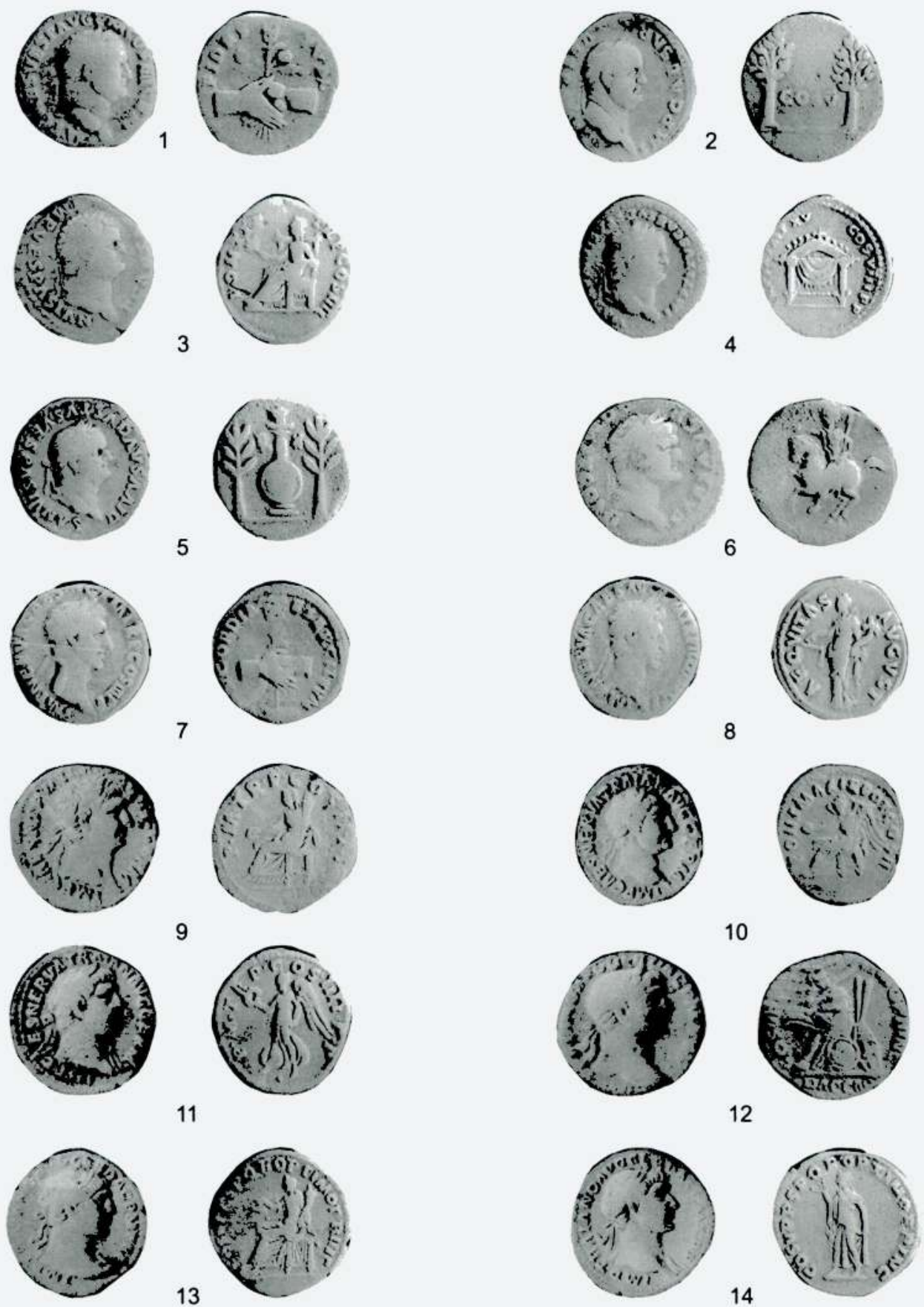


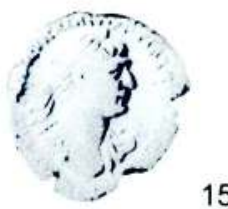

15

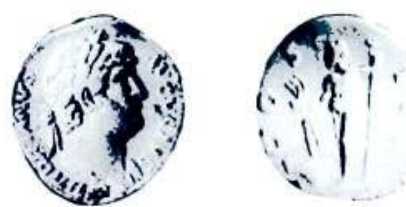

17
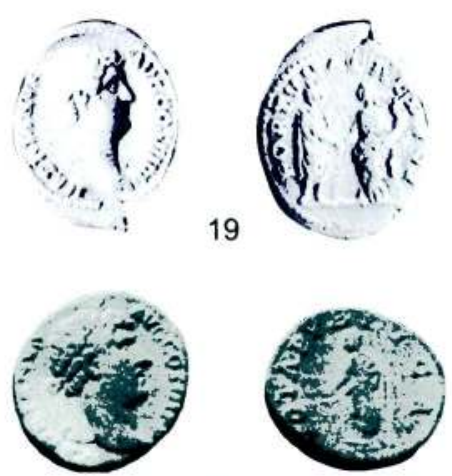

21
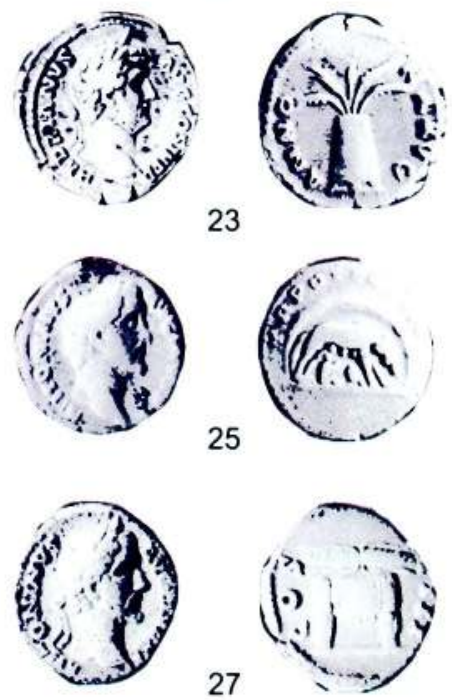
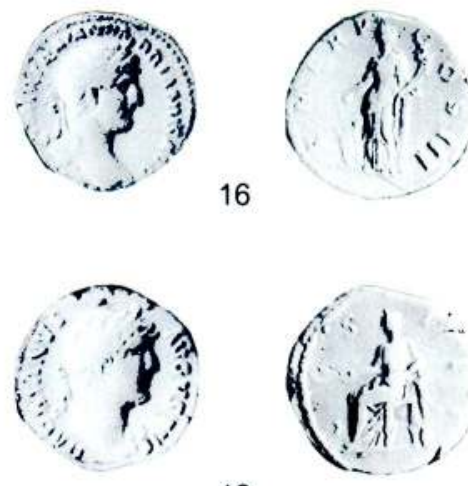

18
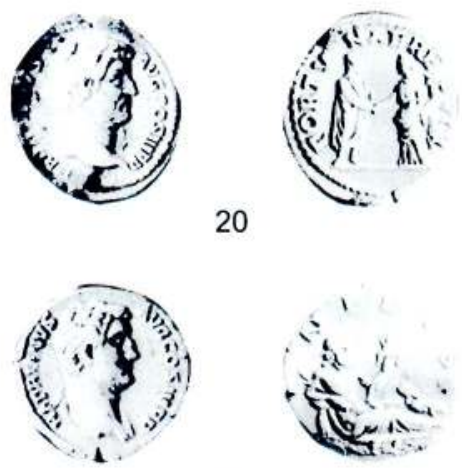

22

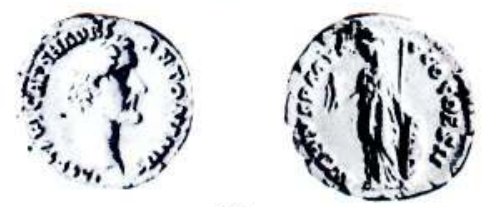

24
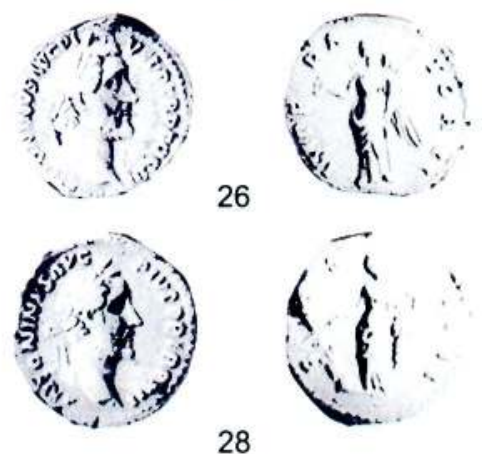
Melih Arslan - Ülkü Devecioğlu

LEVHA III
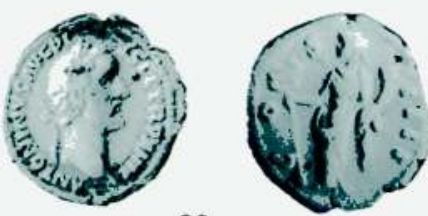

29

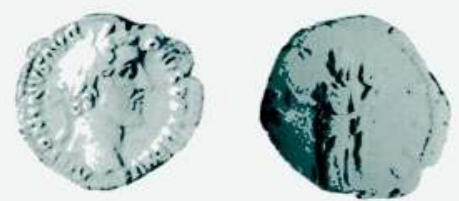

31
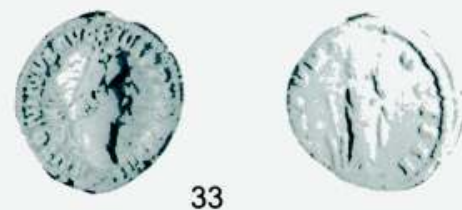

33
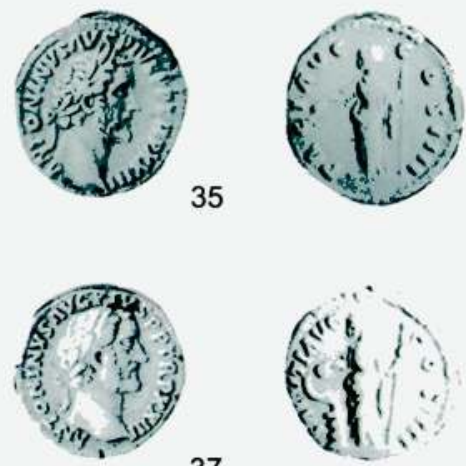

37
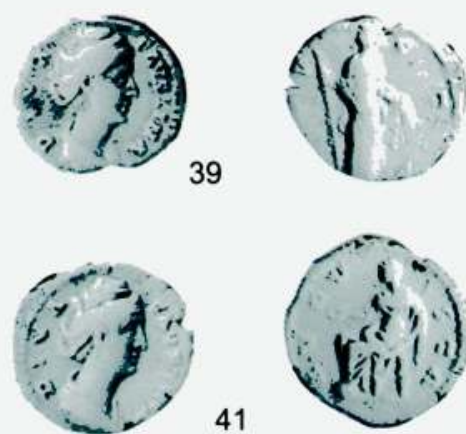
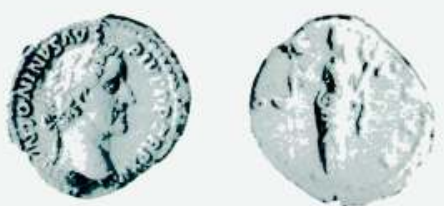

30

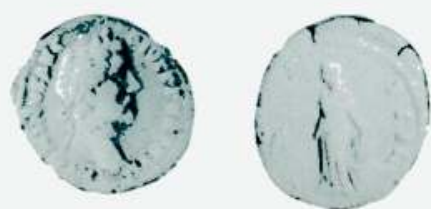

32
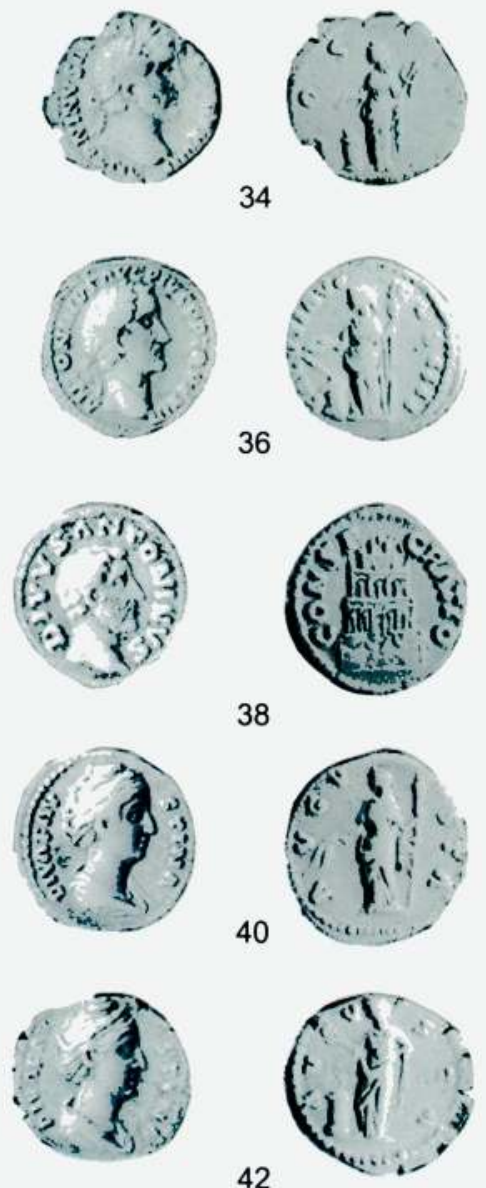
LEVHA IV
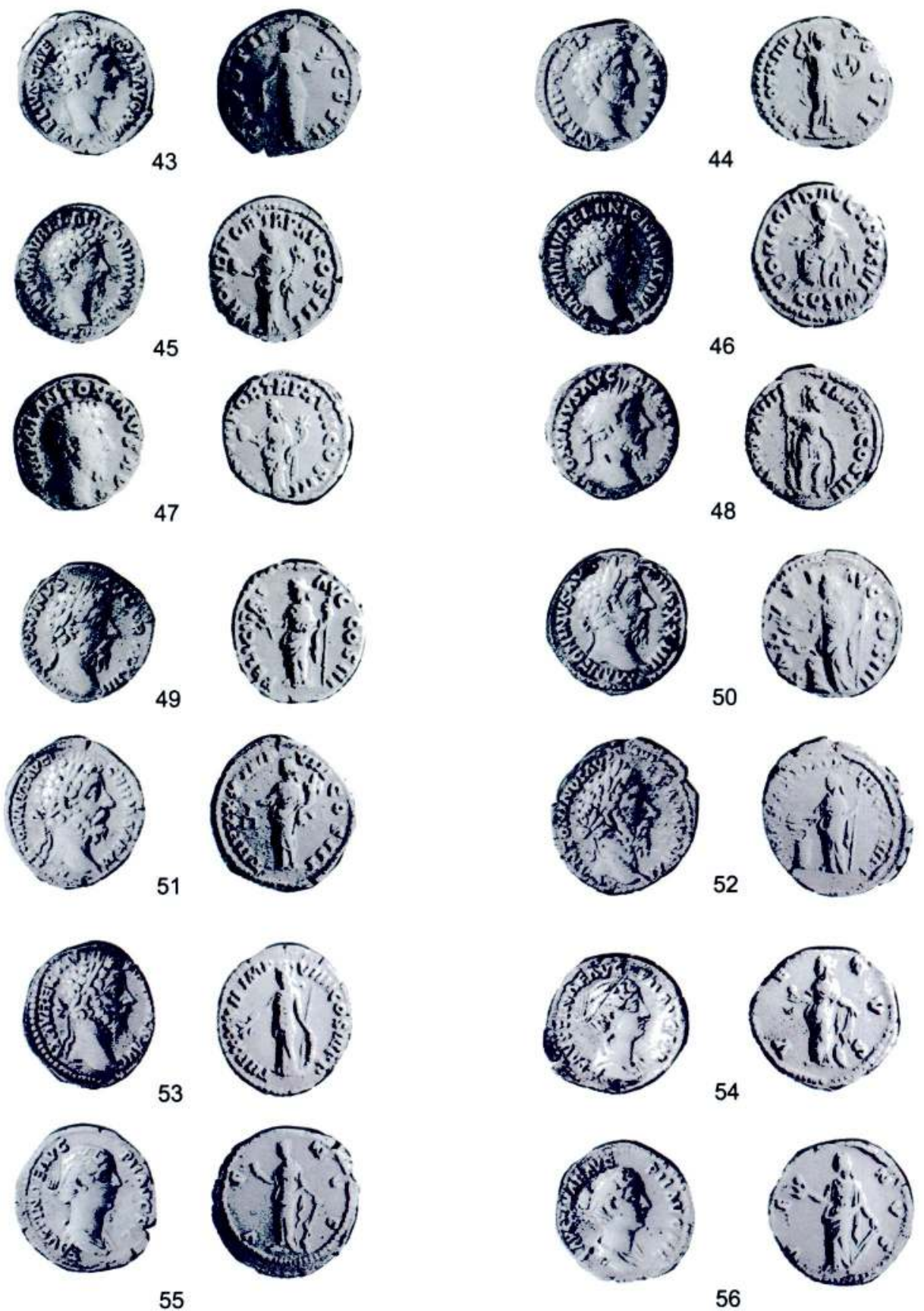

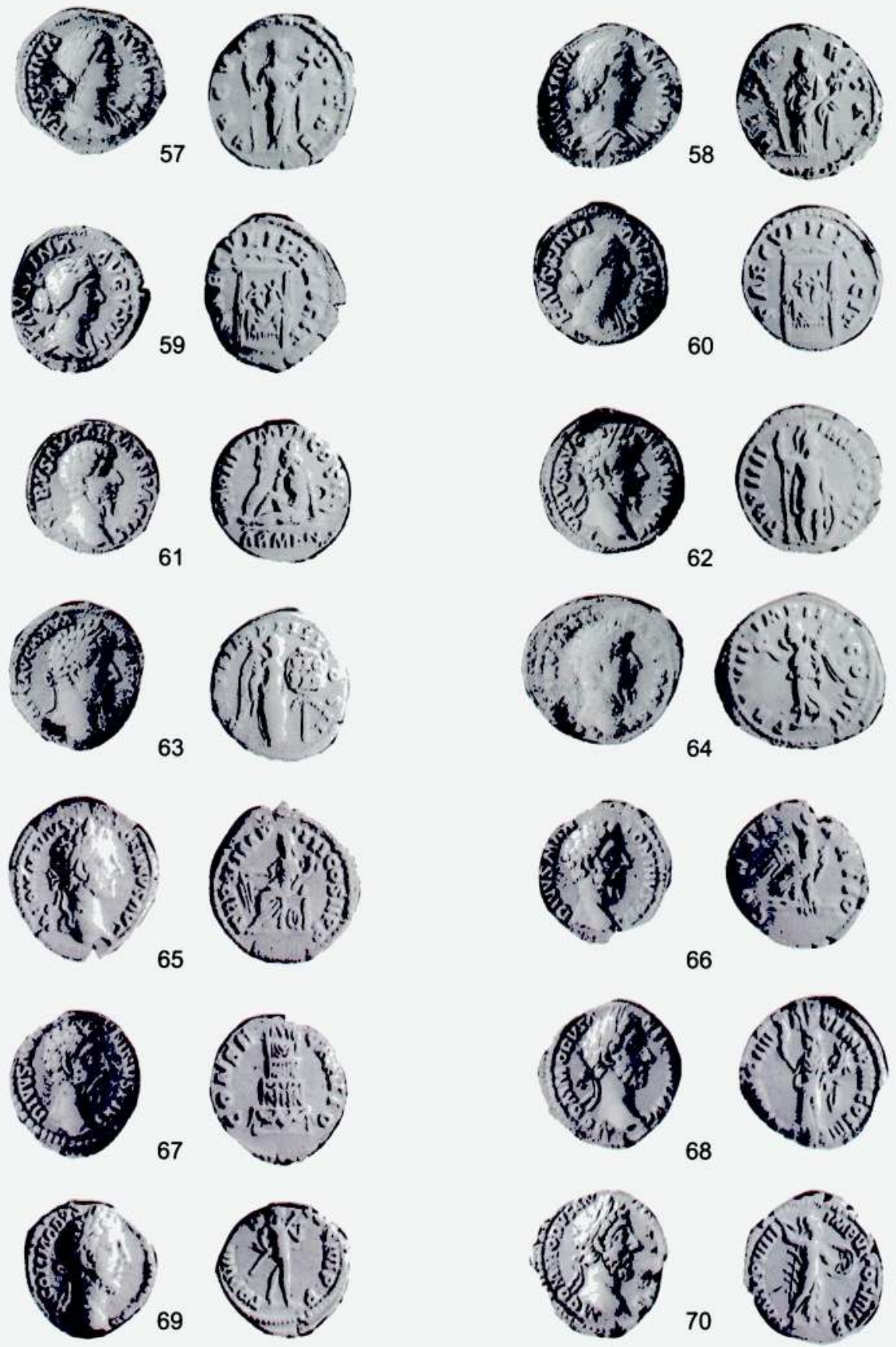

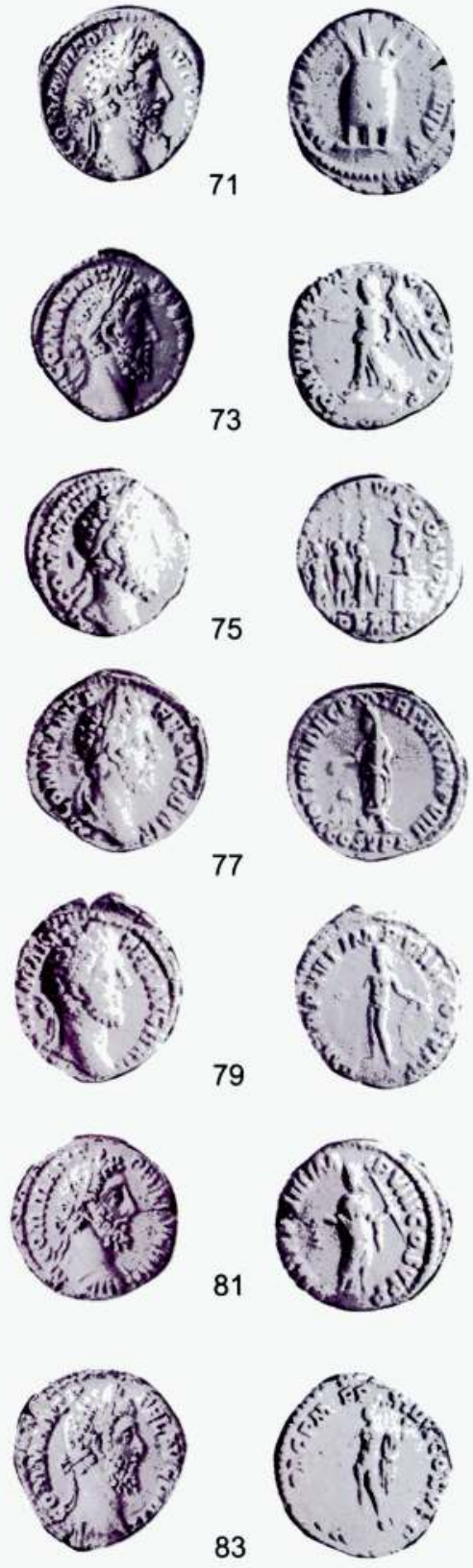
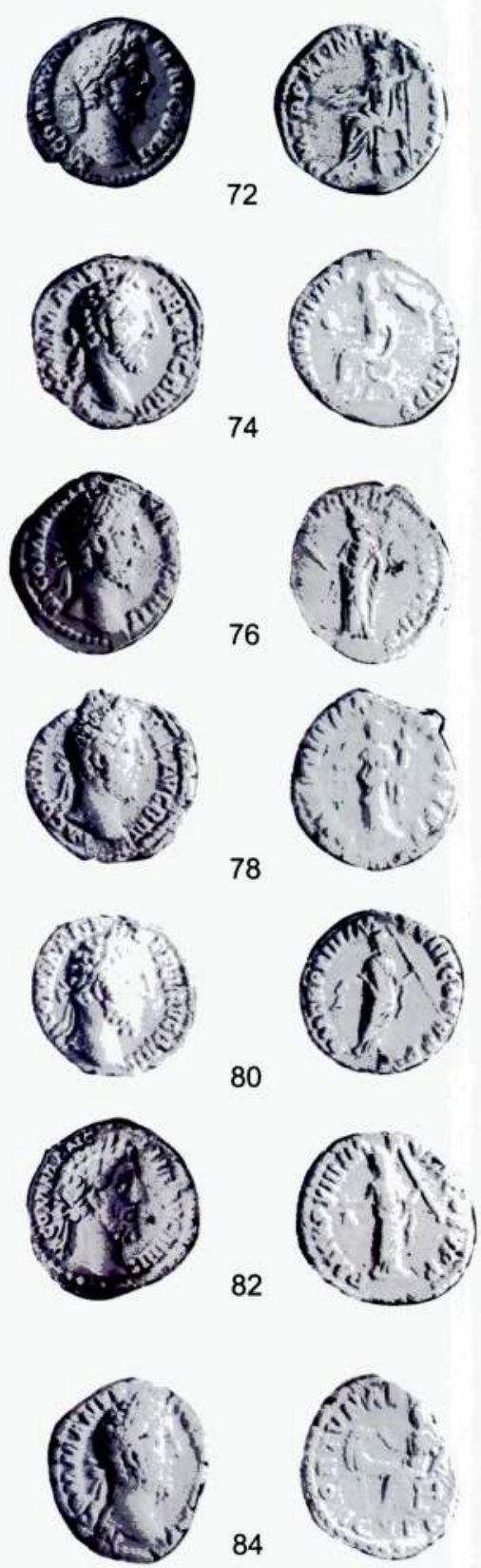

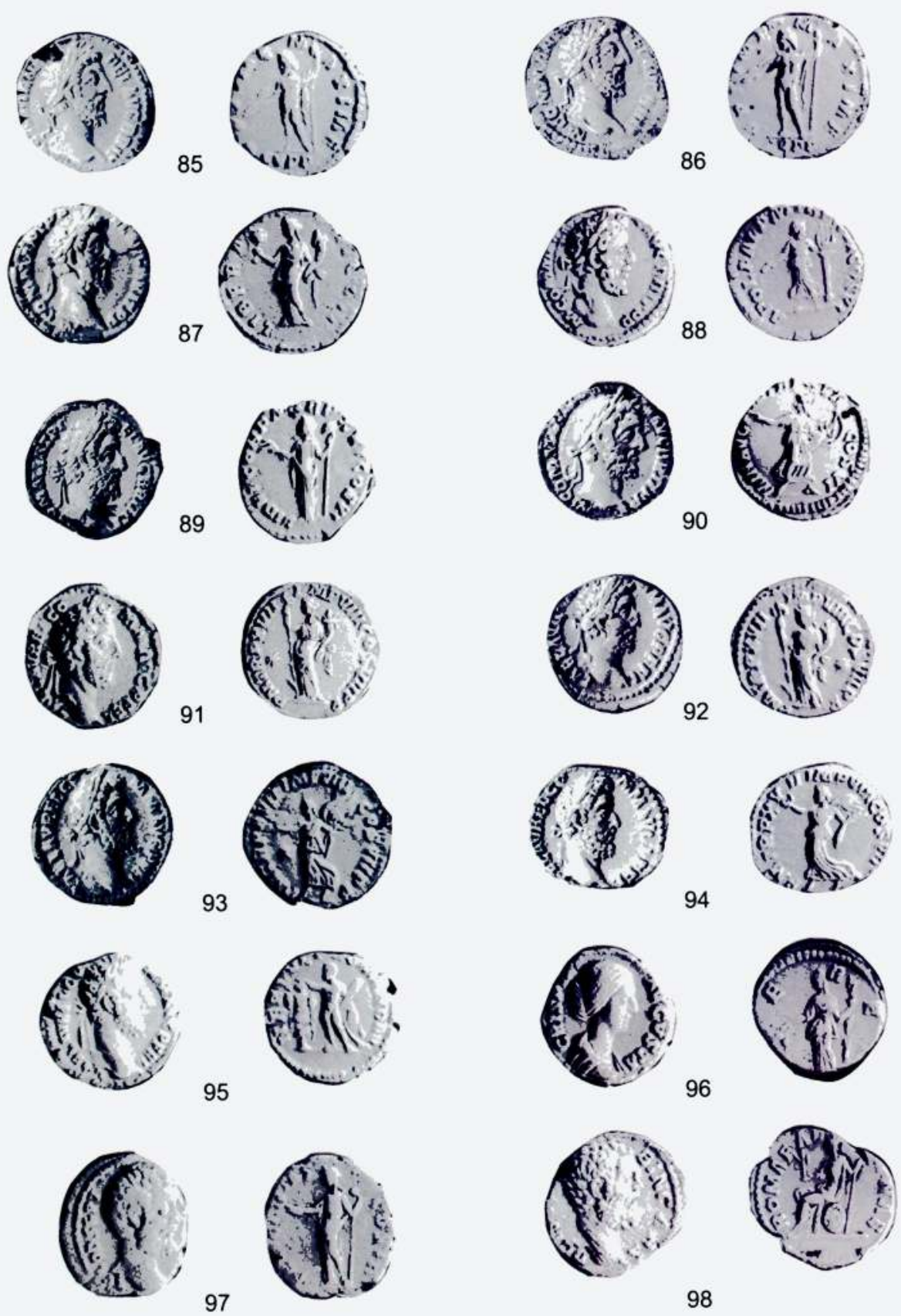


\section{LEVHA VIII}
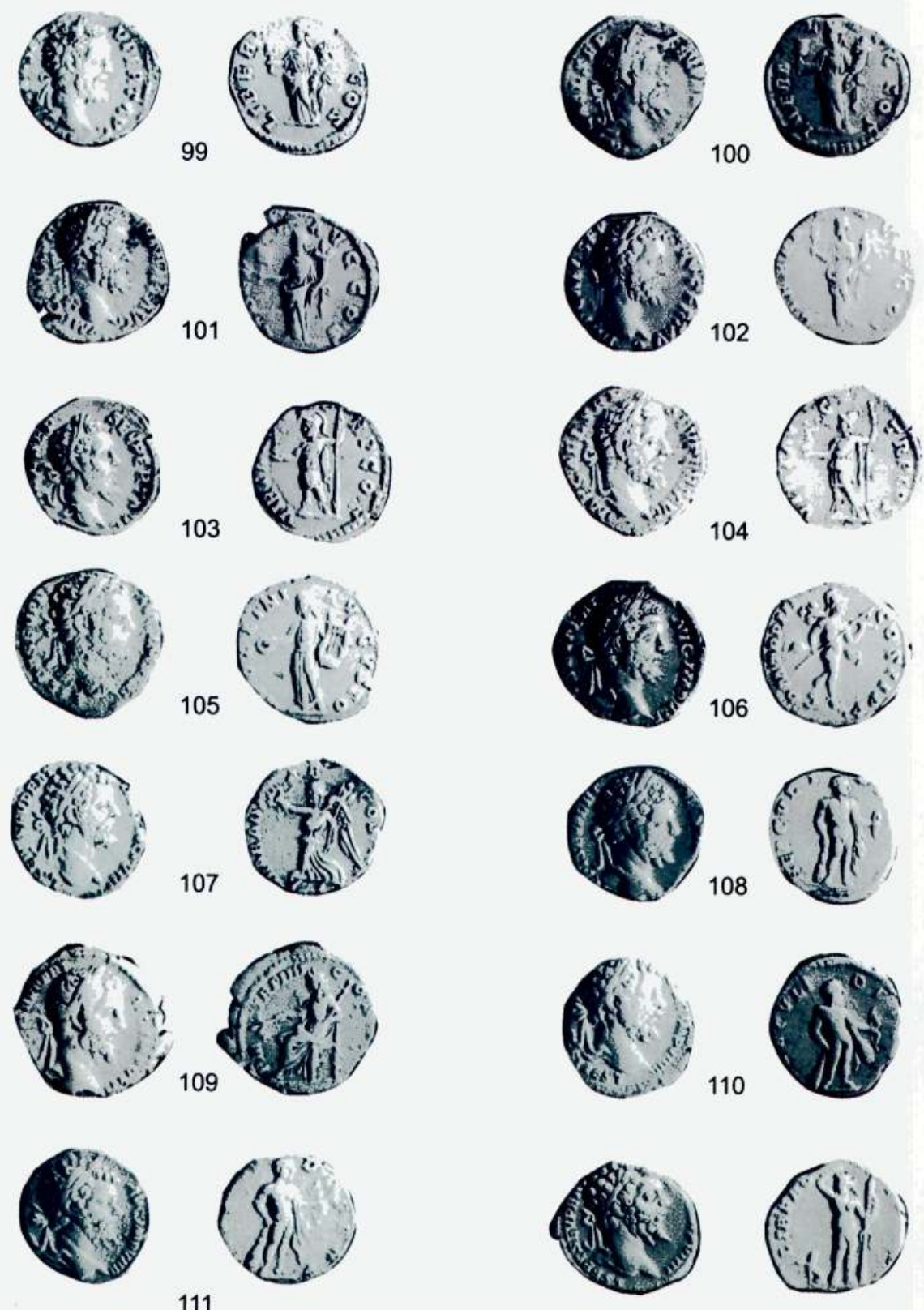

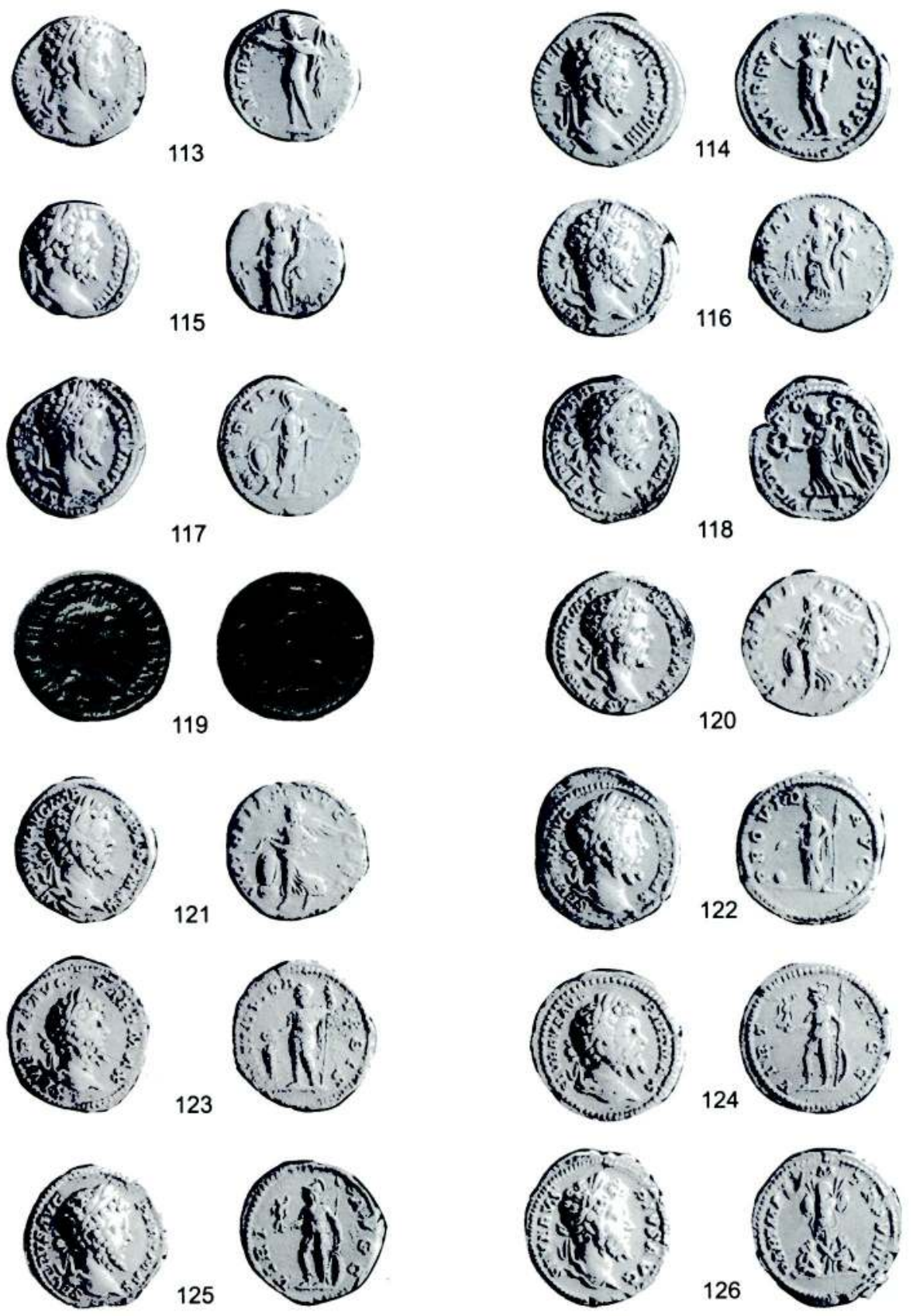
LEVHAX

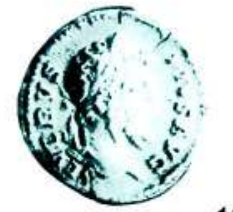

127
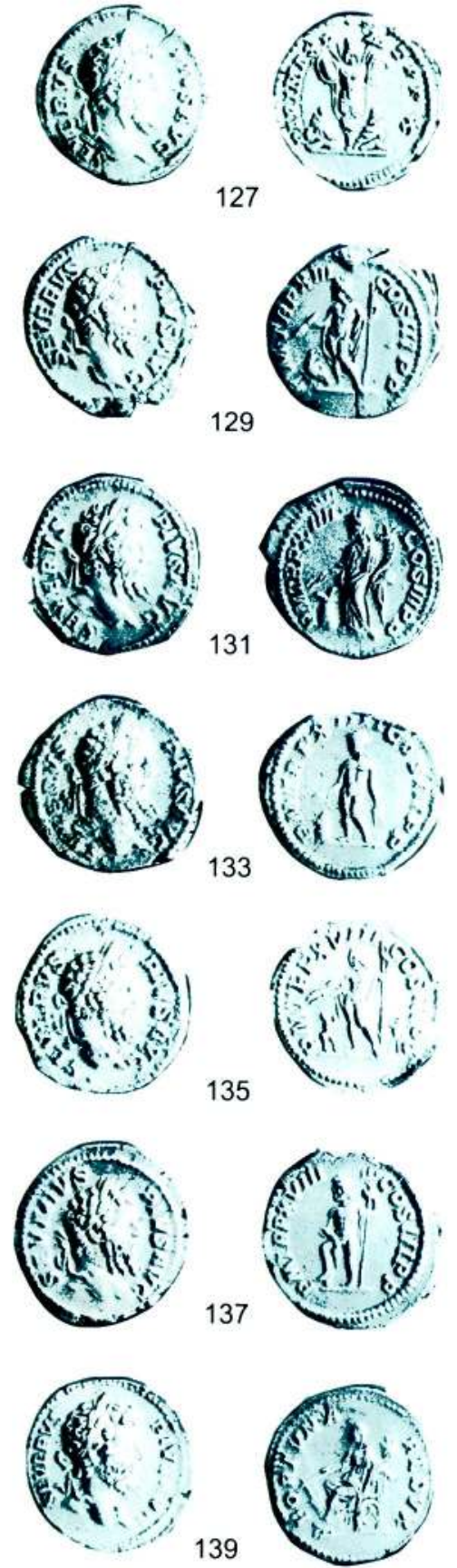
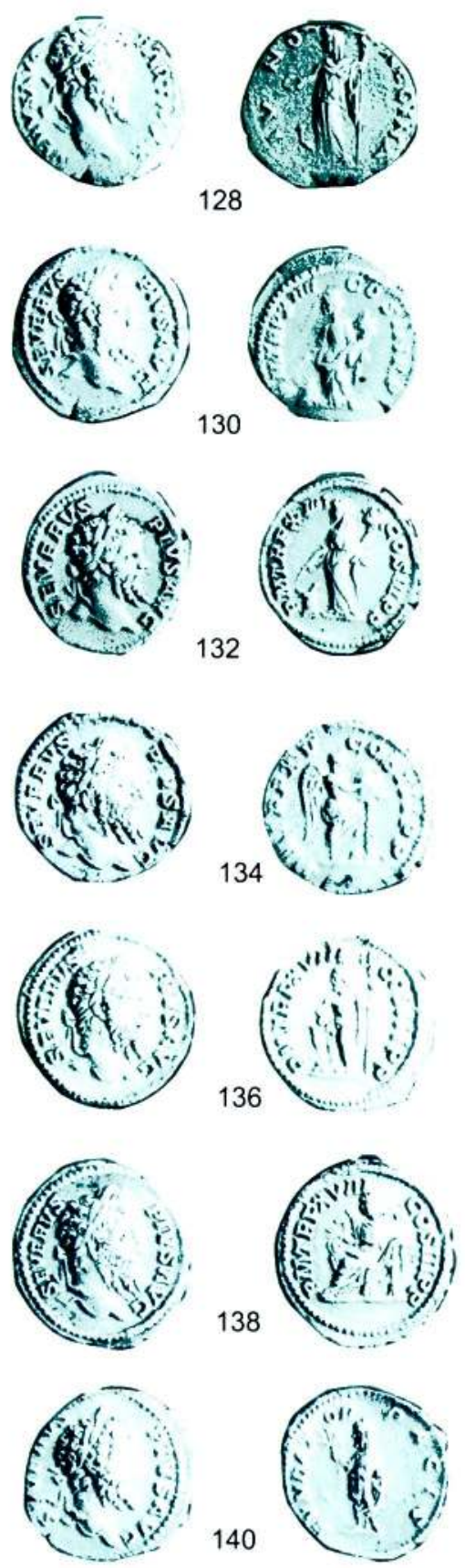
Melih Arslan - Ülkü Devecioğlu

LEVHA XI
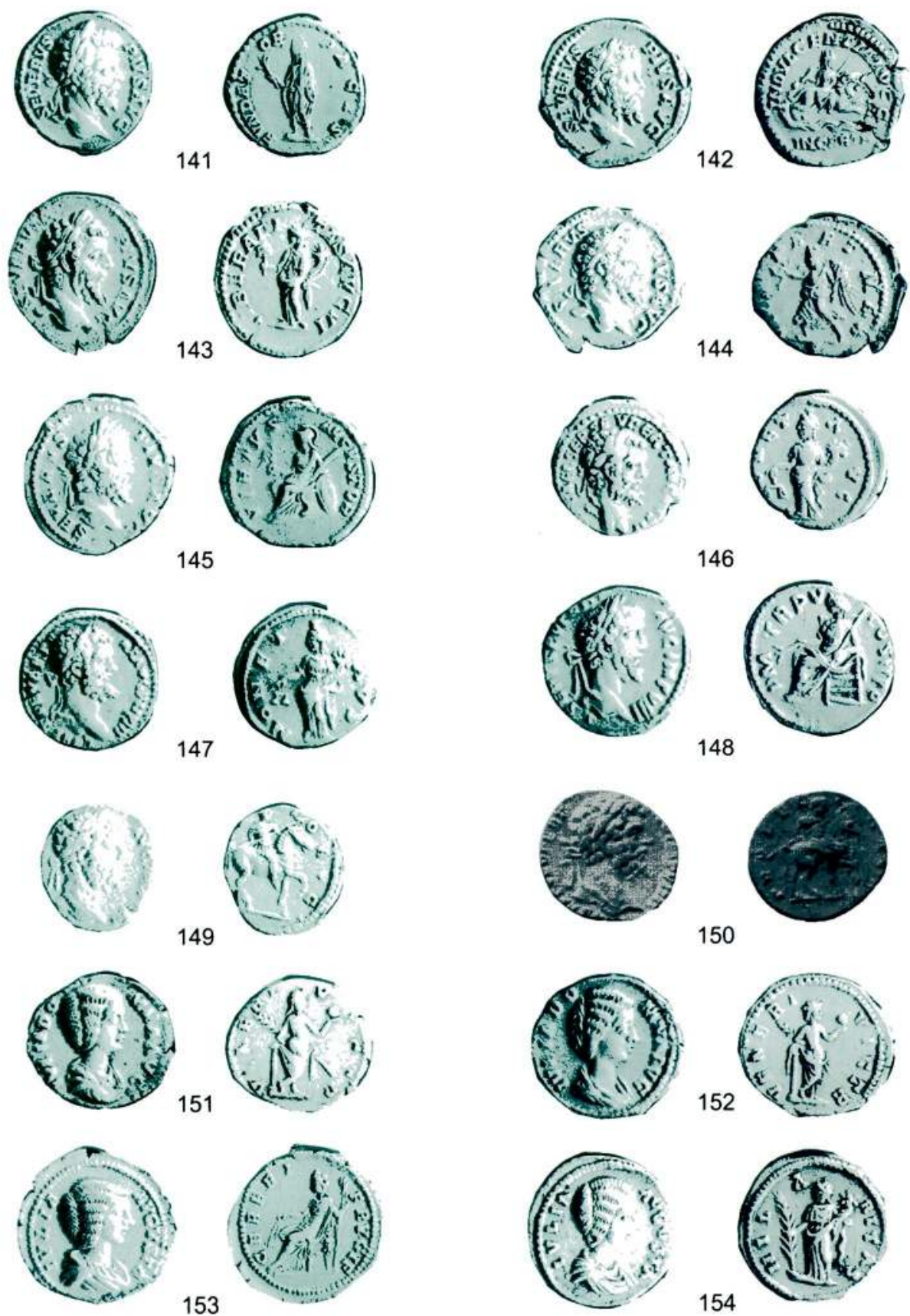

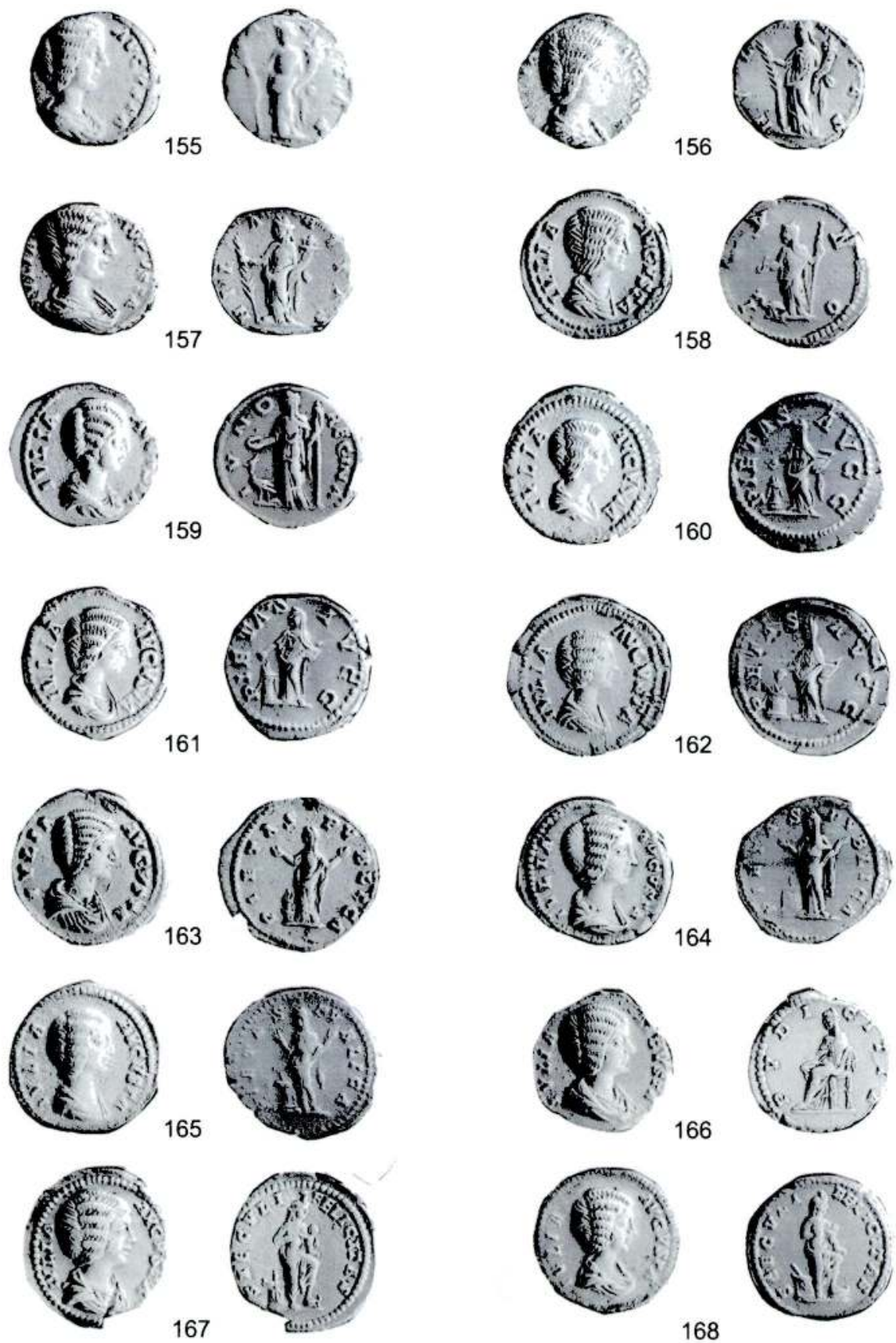
Melih Arslan - Ülkü Devecioğlu

LEVHA XIII
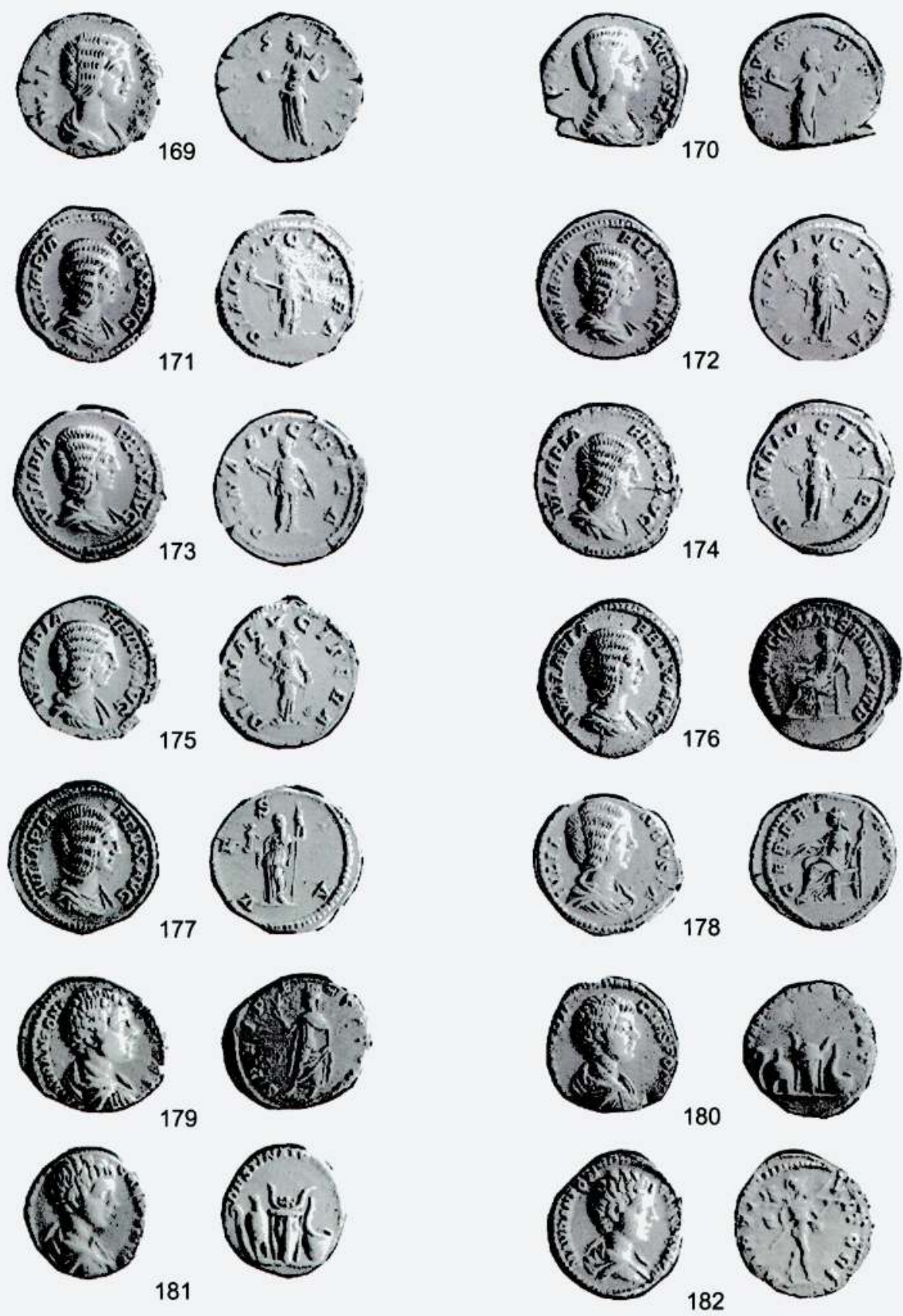

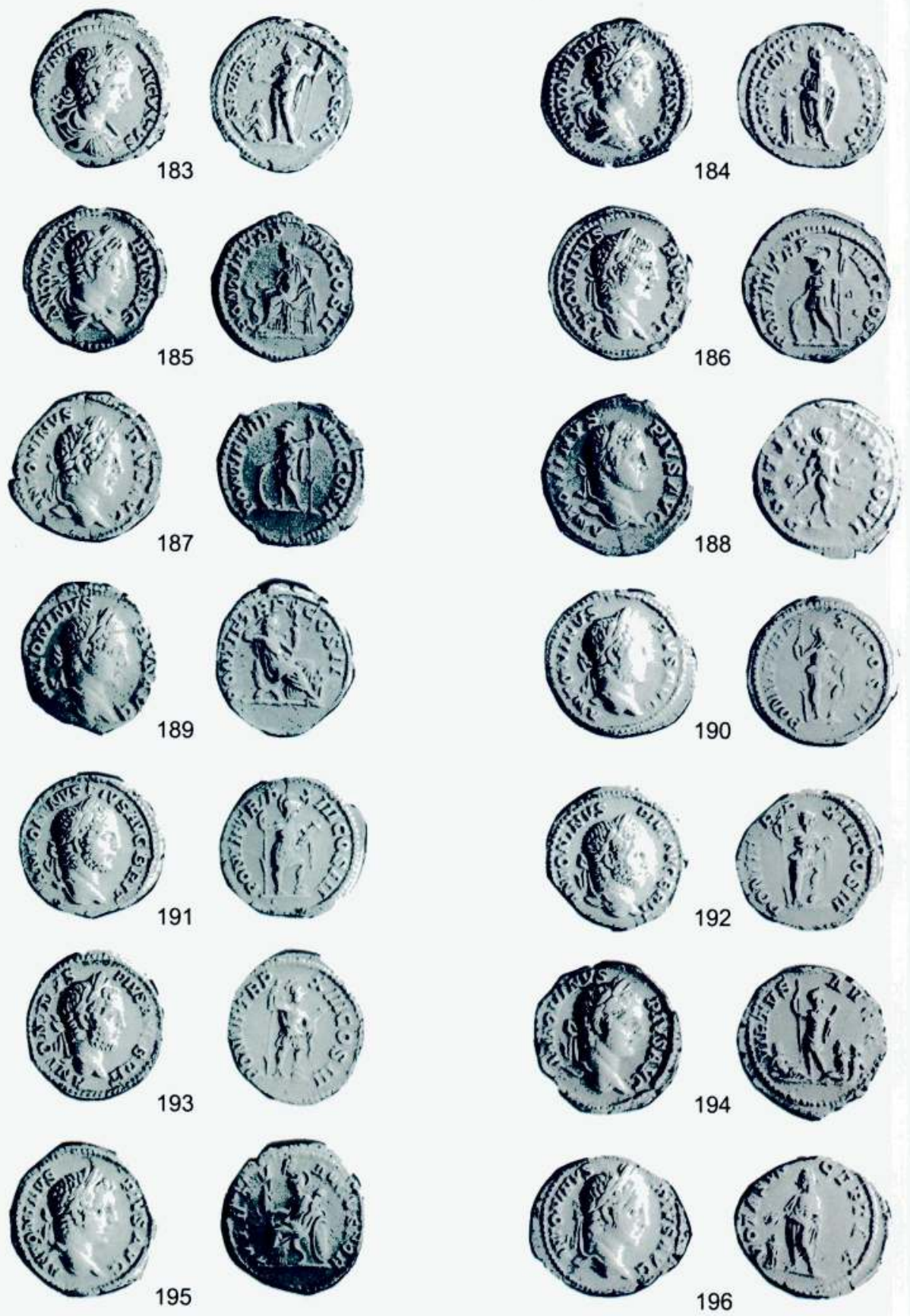

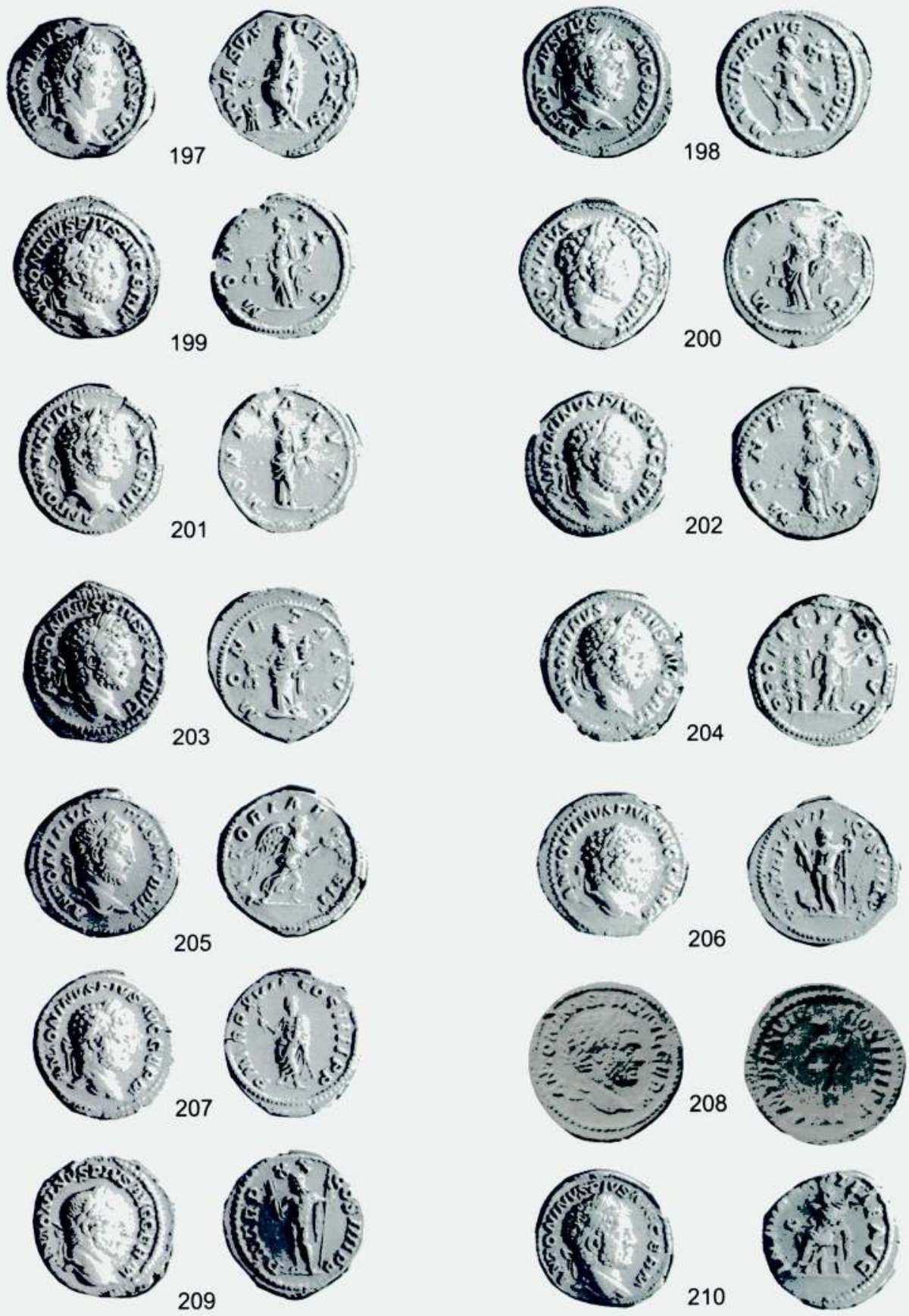
LEVHAXVI

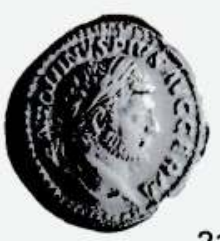

211
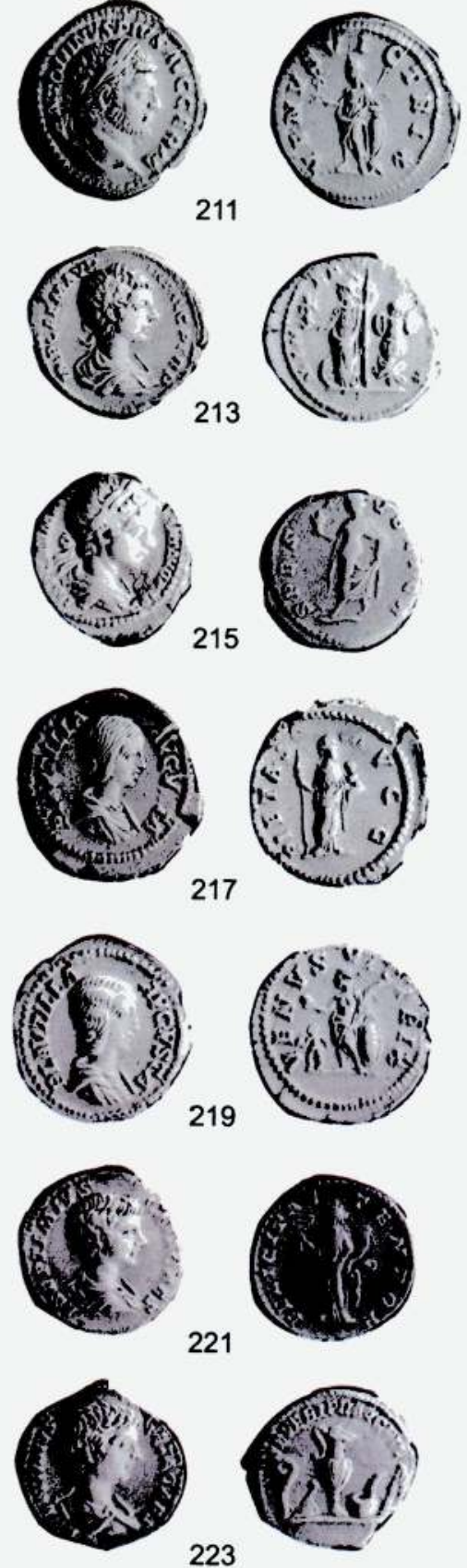
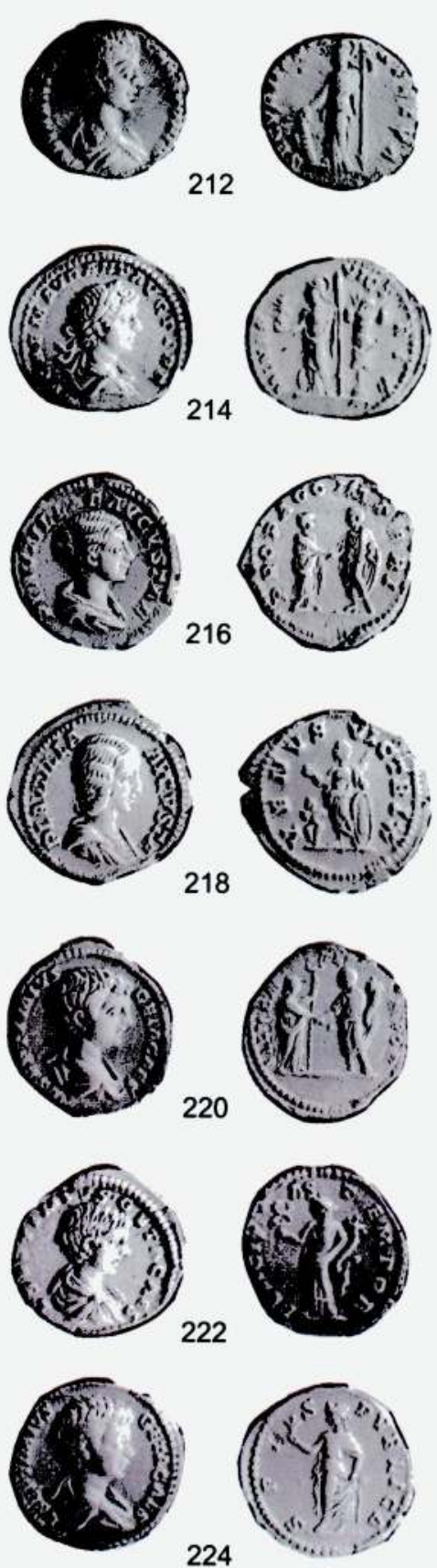

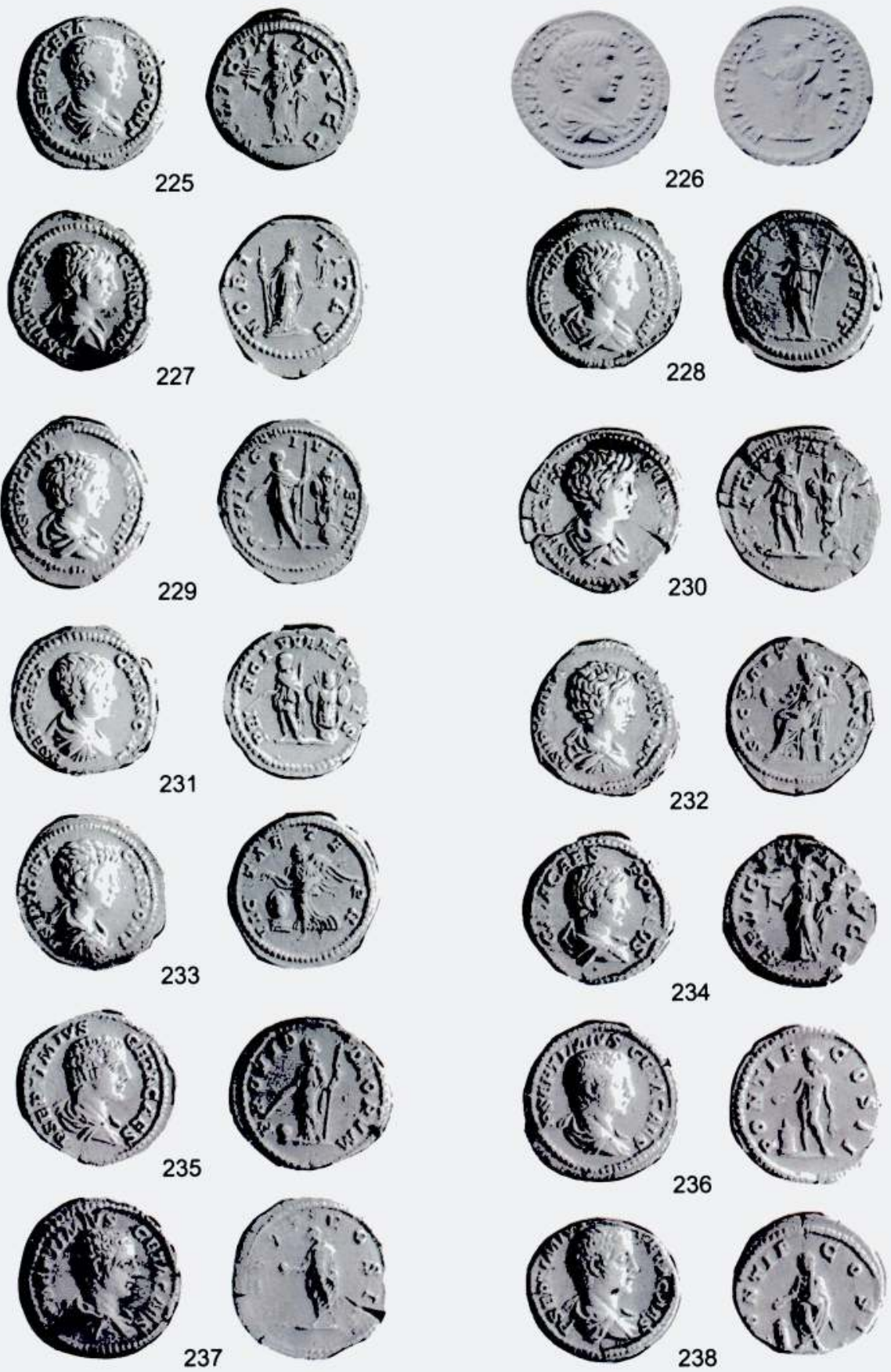
LEVHA XVIII
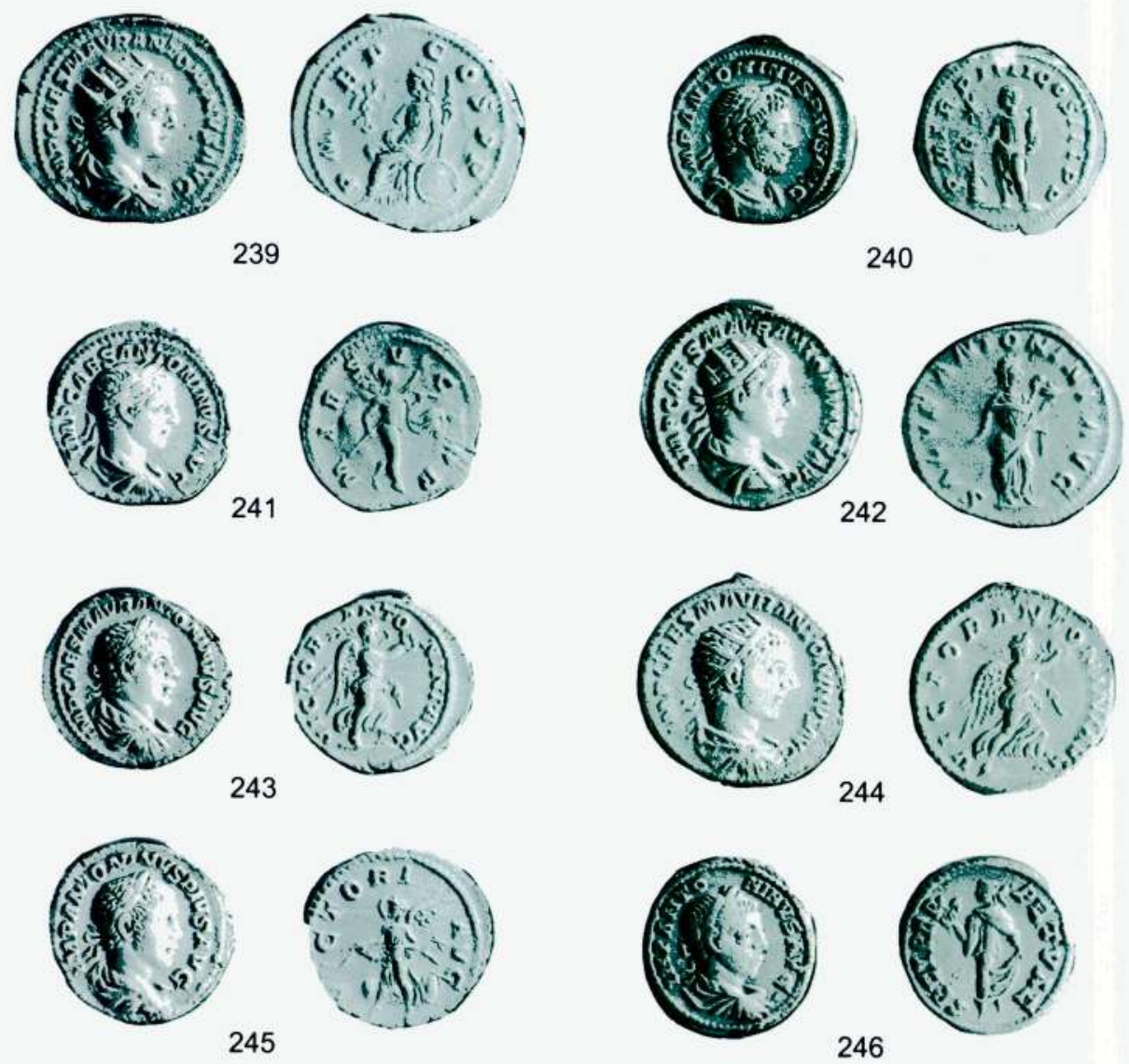